\title{
La nouvelle Europe politique des camps d'exilés : genèse d'une source élitaire de phobie et de répression des étrangers
}

Jérôme Valluy

\section{(2) OpenEdition}

Journals

Édition électronique

URL : http://journals.openedition.org/conflits/1726

DOI : $10.4000 /$ conflits. 1726

ISSN : $1777-5345$

Éditeur :

CCLS - Centre d'études sur les conflits lilberté et sécurité, L'Harmattan

\section{Édition imprimée}

Date de publication : 1 mars 2005

Pagination : 13-69

ISBN : 2-7475-8103-9

ISSN : 1157-996X

\section{Référence électronique}

Jérôme Valluy, «La nouvelle Europe politique des camps d'exilés : genèse d'une source élitaire de phobie et de répression des étrangers ", Cultures \& Conflits [En ligne], 57 | printemps 2005, mis en ligne le 06 janvier 2010, consulté le 30 mars 2021. URL : http://journals.openedition.org/conflits/1726 ; DOI : https://doi.org/10.4000/conflits. 1726

Ce document a été généré automatiquement le 30 mars 2021.

Creative Commons License 


\title{
La nouvelle Europe politique des camps d'exilés ${ }^{1}$ : genèse d'une source élitaire de phobie et de répression des étrangers
}

\author{
Jérôme Valluy
}

1 Les projets dits d'externalisation de l'asile sont apparus fin 2002 dans les débats européens, pour désigner des politiques tendant à délocaliser dans des camps placés hors de l'Union européenne les procédures d'examen des demandes d'asile ainsi que l'accueil des demandeurs d'asile et des réfugiés. Elaborés par des gouvernements européens en collaboration avec le Haut-Commissariat aux Réfugiés de l'ONU (HCR) et la Commission européenne, ces projets visent à créer des «zones de protection spéciale » dans certaines régions du monde (Afrique centrale, Moyen-Orient...) afin d'y concentrer les réfugiés et d'éviter ainsi leur migration vers les pays européens. Il s'agit aussi de créer au bord de l'Europe des «centres» pour y transporter et enfermer durant le traitement des demandes d'asile les exilés approchant des territoires européens. Rendus publics peu avant le Sommet européen de Thessalonique (juin 2003) et réactualisés durant l'été 2004, ces projets ont fait scandale, certains s'inquiétant d'une dérive politique historique.

2 Il s'agit d'apporter à ces débats d'une actualité brûlante, un point de vue sociologique, distancié et éventuellement décalé par rapport aux enjeux les plus immédiats de cette actualité. En amont d'observations juridiques ou anthropologiques relatives aux camps d'enfermement et de regroupement forcé issus de ces projets politiques, il s'agit de comprendre quels sont les facteurs sociaux qui rendent possibles l'élaboration technique et l'expression politique de tels projets, d'analyser la genèse de ces politiques, afin de bien en saisir les caractéristiques et la portée. On se situera ainsi également en amont des jugements de valeur et, chronologiquement, de l'actualité immédiate pour tenter d'identifier les origines des représentations sociales et des processus de décision qui façonnent ces politiques européennes. Deux questions 
connexes seront examinées : comment passe-t-on en moins de trente ans de la situation prévalant encore dans les années 1960 où les exilés ${ }^{2}$ étaient perçus comme des victimes ${ }^{3}$, objets de compassion, à celle de coupables à chasser et à enfermer dans des camps? Dans cette transformation des cultures politiques européennes, quelles relations existent entre l'opinion publique et les actions des élites dirigeantes, notamment politiques et administratives, qui participent aux politiques publiques concernées?

3 Si ces questions se posent, c'est notamment parce que les préambules de textes législatifs, les échanges administratifs qui les préparent et les discours ministériels ou partisans qui les évoquent publiquement mettent en avant certaines réponses généralement relayées par les mass-medias et devenues politiquement dominantes : les évolutions culturelles et politiques observables en Europe et, plus largement, en occident proviendraient d'une anxiété croissante des opinions publiques - parfois sous l'effet d'incitations populistes - face à des migrations changeant de nature (économiques plus que politiques), d'ampleur (croissante sous l'attraction d'un Eldorado européen) et de portée (risques de submersion de sociétés occidentales déjà affaiblies par la crise économique, le chômage et les difficultés de financement des services sociaux...). Les nouvelles politiques publiques résulteraient ainsi d'évolutions des opinions publiques et se justifieraient par le souci d'en tenir compte soit pour satisfaire aux exigences de la démocratie représentative soit pour préserver celle-ci d'éventuelles dérives populistes.

4 Cette présentation des réalités sociales soulève un certain nombre de difficultés. Tout d'abord elle n'est pas corroborée par ce que nous apprennent les études sociologiques des processus de décision politique ${ }^{4}$. Ces études font apparaître, de manière récurrente, le rôle primordial de spécialistes de tel ou tel problème public dont les activités professionnelles ou militantes (fonctionnaires, ministres, experts, lobbyistes...) sont pour le moins distantes de l'opinion publique et largement autonomes par rapport à la vie politique telle qu'elle apparaît (médiatique, partisane, électorale...) aux yeux d'un public élargi. La relation, précédemment alléguée au sujet de l'immigration, entre ces deux dimensions de la vie politique - celle des spécialistes et celle de l'opinion publique - demande donc à être contrôlée, notamment en scrutant attentivement la chronologie des faits relevant de l'une et l'autre dimension et en s'assurant ainsi de l'ordre dans lequel interviennent les causes supposées (inquiétudes des opinions publiques) et effets allégués (processus de décision politique, actions publiques).

5 Cette démarche analytique trouve une raison d'être supplémentaire dans une observation, tout aussi récurrente, produite par ces mêmes recherches en sciences sociales $^{5}$ : un problème public qu'il soit énoncé dans l'une ou l'autre des deux dimensions de la vie politique, n'est jamais le simple reflet d'une réalité objective, mais toujours et nécessairement le résultat d'un processus social, incluant luttes et concurrences politiques, de production des données et des interprétations relatives à la réalité sociale. Cette découverte n'interdit pas de rechercher une « vérité objective » de la réalité sociale, elle suggère seulement de prêter une attention soutenue aux écarts éventuels entre celle-ci et les représentations sociales qui s'y rapportent, notamment dans le champ politique, et de s'intéresser particulièrement aux conditions d'émergence de ces représentations sociales dans la société.

6 A cet égard, les changements de nature, d'ampleur et de portée aujourd'hui si couramment imputés aux migrations incitent fortement à l'adoption d'une telle 
approche constructiviste: la théorie d'une migration devenue essentiellement économique occulte la croissance du nombre de réfugiés reconnus comme tels dans le monde depuis trente ans ${ }^{6}$. Après vingt-cinq ans de stabilité autour de 2,5 millions (de 1950 au milieu des années 1970), cette population augmente régulièrement jusqu'à 18 millions en 1992 et 12 millions aujourd'hui. Encore ne s'agit-il que de chiffres relatifs aux réfugiés officiels. Si l'on y ajoute les déplacés internes et les exilés qui n'accèdent pas à ce statut, le nombre de personnes contraintes à l'exil est estimé à près de 50 millions. Or les causes initiales de migrations forcées ne sont pas mystérieuses. Pour n'en citer que quelques unes ${ }^{7}$ : dans la Corne de l'Afrique une série de guerres déplace des millions de réfugiés. Le long conflit afghan en fait fuir six millions. En Amérique centrale, trois guerres déplacent plus de deux millions de personnes. Dans le même temps des Sri Lankais se réfugient en Inde, des Ougandais au sud du Soudan, des Angolais en Zambie et au Zaïre, des Mozambicains dans six pays voisins, etc. Et aux guerres s'ajoute encore la permanence des persécutions politiques, religieuses, ethniques et sociales dans le monde ${ }^{8}$.

7 La théorie d'une hyper-attractivité des pays riches comme principal facteur d'exil résiste mal à l'observation des évolutions de populations réfugiées dans les différentes régions du monde ${ }^{9}$. En ordre de grandeur, pour les vingt-cinq dernières années, le chiffre fluctue autour de 1 million sur le continent américain; il passe de 500000 à 3 millions sur le continent européen, l'essentiel de la croissance ayant lieu en 1991/1992 ; mais il passe de 2 à 7 millions en Afrique, en augmentation depuis 1985 et de 1 à 8,5 millions en Asie, l'essentiel de l'accroissement ayant lieu en 1980 et 1981. L'évolution en Europe est donc postérieure et très inférieure à celles de l'Asie et de l'Afrique. Elle implique en effet des personnes fuyant d'abord à proximité de leur patrie puis trouvant ensuite refuge dans une contrée plus éloignée. Sur les dizaines de millions d'exilés dans le monde quelques millions sollicitent finalement l'asile dans les pays occidentaux où les réfugiés représentent, en tendance, moins de $1 \%$ de la population. Pas plus de huit de ces pays ${ }^{10}$ apparaissent dans la liste des quarante premiers pays d'accueil.

8 L'interprétation en termes d'envahissement nécessite de considérer non pas les réfugiés mais les demandes d'asile. Elle sont stables depuis vingt-cinq ans en Amérique du Nord, Australie et Nouvelle-Zélande ${ }^{11}$. En Europe ${ }^{12}$, au contraire, ce chiffre annuel se situait entre 100000 et 200000 jusqu'en 1988. Il augmente jusqu'à près de 700000 en 1992 et redescend à un peu plus de 250000 en 1995. Depuis lors, une nouvelle croissance est observable jusqu'à 450000 en 1999 suivie d'une décroissance ramenant à 325000 en 2003. On peut certes souligner un écart entre les continents. Mais l'on peut remarquer aussi que durant les dix dernières années, la tendance en Europe est stable, avec des fluctuations de faible amplitude, autour du niveau pivot de 400 000. En outre, si l'on rapporte ces chiffres aux nombres annuels de naissances nouvelles : le ratio est de l'ordre de $10 \%$, ses valeurs successives variant faiblement autour de ce niveau. Enfin, l'ensemble de ces données ne tient pas compte des disparitions d'exilés que ce soit par décès ou par départ. En France entre le 31 décembre 1973 et le 31 décembre 1993, ces "sorties» représentent pas moins de $80,67 \%$ des «entrées» de la population des réfugiés ${ }^{13}$ ! Il n'existe pas de données pour les demandeurs d'asile déboutés de leur demande, leurs "sorties » sont donc toujours ignorées mais on peut les supposer au moins aussi importantes sinon plus que celles des réfugiés statutaires.

9 Le rappel de ces données fait apparaître l'intérêt d'une démarche constructiviste en tant qu'elle incite à s'interroger sur la genèse des représentations sociales et des 
politiques publiques relatives aux exilés. A cet égard il faut rappeler le poids du passé dans l'action des Etats et sa dépendance a des trajectoires de longue durée ${ }^{14}$. De ce point de vue sociologique, constructiviste et historique, la "politique des camps", telle qu'elle est analysée dans la troisième partie de cet article, ne peut pas émerger en septembre 2002 comme une "génération spontanée " hors de tout contexte. Notre hypothèse est au contraire celle d'un enchaînement de trois processus historiques distinguables mais étroitement interdépendants, à travers lesquels s'opère dans la culture politique d'élites dirigeantes européennes, technocratiques notamment, une mutation qui rend possible et, probablement, difficilement réversible l'avènement d'une politique des camps. Dans un premier temps, la stigmatisation de l'exilé comme faux-réfugié est un résultat du rejet progressif de la quasi-totalité des demandes d'asile. Or cette évolution administrative s'amorce bien avant que la figure du faux-réfugié n'envahisse l'espace public médiatique (La spirale du rejet). Puis, l'exilé se trouve érigé en menace au fur et à mesure que prospèrent les idées nationalistes et sécuritaires. Or cette menace est visée dans des actes de gouvernement bien avant d'être désignée dans l'espace public électoral par les partis d'extrême droite (le tournant nationalsécuritaire). Enfin, la banalisation des stratégies de dissuasion des exilés notamment par bannissement et enfermement est alors possible. Or leur gestation s'opère d'abord dans des cercles technocratiques avant d'émerger dans l'espace public des déclarations ministérielles (La politique des camps) ${ }^{15}$.

\section{La spirale du rejet, stigmatisation du faux-réfugié}

10 Lorsque l'on relit en 2005 l'histoire des exilés d'abord chiliens puis vietnamiens, cambodgiens et laotiens durant les années $1970^{16}$ on est frappé par l'absence de doutes exprimés à l'époque quand à leurs motifs de départ. Trente ans après, dans tous les pays occidentaux, la situation est diamétralement inversée : ceux qui demandent l'asile aujourd'hui sont à peine écoutés et leurs récits de vies, dans leur très grande majorité ne sont pas crus, quelles que soient leurs nationalités et les situations fuies.

11 L'image du demandeur d'asile, faux-réfugié inventant son histoire de persécution pour trouver du travail, est devenue une évidence indiscutée au vu des taux de rejet des demandes d'asile, entre $80 \%$ et $100 \%$ en Europe. D'un autre point de vue, peut-être affecté par une expérience personnelle de juge de la demande d'asile ${ }^{17}$, le plus frappant est justement le caractère indiscuté tant de cette croyance que des modalités administratives et juridictionnelles de production d'une statistique idéologiquement aussi fondamentale.

Dans tous les pays européens, les taux de rejet commencent à s'élever dès les années soixante-dix c'est-à-dire longtemps avant que l'enjeu ne soit médiatisé et politisé dans l'espace public. Configurées par les lois et décrets de politiques diplomatiques et policières, les institutions de l'asile augmentent leurs rejets jusqu'aux maximums actuels : cette dimension du processus est essentiellement technocratique. Au cours des années 1980, l'enjeu émerge dans l'espace public avec diverses formes d'hostilité à l'égard des exilés ${ }^{18}$. Les taux de rejet font alors l'objet d'usages politiques qui, aujourd'hui encore, alimentent les discours sur l'invasion des faux-réfugiés. En réaction à ces discours qu'elles ont elles-mêmes alimentées, les autorités gouvernementales, administratives et juridictionnelles affichent leur sévérité en restreignant la définition du réfugié, augmentant d'autant les taux de rejet. Ce processus circulaire entraîne une 
radicalisation des rhétoriques du rejet à la fois dans la sphère technocratique et dans l'espace public.

\section{Le rejet technocratique des demandes d'asile}

13 La spirale du rejet s'amorce avec une histoire : le demandeur d'asile raconte ce qui l'a conduit à quitter son pays et cette relation écrite ou orale est difficile et, nécessairement, longue ${ }^{19}$. Cela ne tient pas seulement à des besoins psychologiques comme celui de verbaliser une expérience choquante pour en atténuer les séquelles traumatiques ou d'être reconnu comme victime innocente d'une persécution qui stigmatisait un coupable. Les récits s'allongent surtout parce que l'exil est contraint. Or cette contrainte se construit sur plusieurs années. Même l'explication d'un départ hâtif parcourt plusieurs années de vie : soit parce que la situation de l'exilé s'est détériorée insidieusement jusqu'à un stade, subjectivement perçu, de crainte rédhibitoire ; soit parce que le facteur déclencheur aussi prompt soit-il n'économise pas ultérieurement à l'exilé la peine de remonter loin dans le temps pour s'expliquer à lui-même d'abord, et aux autres ensuite, cet étrange basculement de sa vie ; soit enfin parce que la société chaotique qui pousse à l'exil recèle une complexité difficile à maîtriser dans la narration biographique comme dans l'analyse sociologique. Aussi n'est-il pas rare qu'une demande d'asile retrace dix ou vingt années d'une vie et qu'elle enfle sous l'accumulation des faits et des craintes et se complique encore dans la tourmente de l'exil. Chacune de ces vies pourrait remplir un roman et bien des biographies furent publiées à moindre coût humain.

14 Les formulaires des procédures d'asile ne font pas place à une telle expansion et les procédures d'asile ne laissent guère de temps à l'empathie. Les rejets sont massifs, la procédure expéditive et inquisitoriale ${ }^{20}$. Le demandeur s'expose d'abord par écrit et en quelques pages. Le récit est bref, sec, improvisé sur un coin de guichet, sous la pression d'une file d'attente, handicapé par le recours à un intermédiaire, voir dévoyé par celui qui peut traduire, sait écrire et croit savoir ce qu'il vaut mieux noter. Dans certains pays comme la Grèce, et pour une proportion d'exilés déjà considérable en GrandeBretagne $^{21}$ et en Espagne ${ }^{22}$, et croissante dans les autres pays, le rejet est prononcé dès ce stade ; la demande d'asile étant alors qualifiée de «manifestement infondée ». Pour ceux qui bénéficient d'une procédure plus longue commence alors la préparation d'un examen crucial dans des conditions matérielles et psychologiques qui mèneraient bien des étudiants à l'échec. La précarité impose notamment un souci de survie immédiate qui marginalise les autres préoccupations : manger et dormir passe avant la procédure et l'élaboration du récit. Les plus chanceux bénéficient de soutiens associatifs, familiaux ou amicaux. Mais ces soutiens, souvent utiles, peuvent être néfastes par incompétence bienveillante. Bien peu d'exilés ont cette connaissance minimale du droit qui leur serait nécessaire pour apercevoir dans leur propre vie ce qui importe aux yeux de leurs juges. Aucun pays n'assure d'assistance juridique systématique. Les mois voire les années d'attente affectent la mémoire comme chacun peut s'en assurer en tentant de reconstituer son propre passé.

15 Viennent ensuite les phases d'audition, pour certains, déjà minoritaires. Les durées d'auditions vont de quelques minutes à quelques dizaines de minutes suivant les pays et les étapes de la procédure. Plusieurs enquêtes en Belgique ${ }^{23}$, France $^{24}$, Pays-Bas ${ }^{25}$ et au Royaume-Uni ${ }^{26}$ attestent de cette exiguïté temporelle dans laquelle est placée toute 
expression d'une demande d'asile. Ces études présentent le même constat de collision entre les temps d'écoute et la possibilité pour l'exilé de se faire comprendre. Chacun peut en faire l'expérience : prendre sa montre et essayer de se raconter à soi même ou à un tiers les dix ou quinze dernières années de sa propre vie sans dépasser le temps de quelques dizaines de minutes voir même d'une heure. L'expérience peut encore être rapprochée de la réalité procédurale du droit d'asile en déduisant du temps disponible les durées de traduction (en différé, pour des raisons financières) et en l'entrecoupant des multiples questions pointues d'un interrogateur pris dans une logique de suspicion. Encore faudrait-il intégrer à cette expérience les décalages culturels qui affectent la communication entre, par exemple, un jeune berger peuhl de Mauritanie réduit en esclavage depuis son plus jeune âge et un fonctionnaire occidental installé dans les hautes sphères de l'Etat ou fraîchement titulaire d'un Master de droit public international. Sous le poids de telles contraintes, de telles conditions sociales d'expression, le récit d'exil est écrasé, laminé. Sa crédibilité finale, lorsqu'elle advient, reste à expliquer et cette explication pour la sociologie est difficile à apporter ${ }^{27}$.

Comment en est-on arrivé là ? De la manière la plus simple qui soit. L'accueil des exilés n'a jamais été conçu comme une finalité essentielle des Etats-providence, comme une finalité assez importante pour justifier des dépenses conséquentes. Et bien rares sont les forces sociales ou politiques, aujourd'hui comme hier, qui réclament des services publics au bénéfice de non-nationaux. Dès son adoption en 1951, la Convention de Genève sur les réfugiés a été mise en œuvre d'autant plus facilement que, au-delà du contexte géopolitique favorable de la Guerre Froide, elle ne nécessitait aucun engagement immédiat de fonds publics. Une infime proportion des exilés demandait l'asile. La plupart d'entre eux parvenaient à refaire leur vie en résidant dans des pays occidentaux largement ouverts et ne voyaient pas l'utilité d'une procédure complexe qui n'avait pas une grande incidence sur les conditions de vie. Certains préféraient même l'éviter ${ }^{28}$. Les quelques milliers de demandes d'asile déposées dans chaque pays pouvaient donc, sans que personne ne s'en plaigne, faire l'objet d'un traitement expéditif, une instruction administrative superficielle débouchant dans la plupart des cas sur l'octroi du statut de réfugié.

17 C'est ce mode de traitement originel qui va perdurer jusqu'à aujourd'hui : un examen prenant entre quelques minutes et quelques heures, suivant les pays et les étapes de la procédure, par des fonctionnaires ne disposant d'aucun moyen d'investigation délocalisée, le plus souvent sans grands moyens de documentation ni d'actualisation des données sur le pays concerné. Comme l'observe Cynthia Hardy en étudiant le processus d'examen des demandes d'asile au Royaume-Uni, au Danemark et au Canada, il ne s'agit jamais d'une technique d'établissement de la vérité relative aux trajectoires biographiques des réfugiés mais d'un processus de construction d'une réalité spécifique : celle du taux de rejet des demandes d'asile et de ce que laisse supposer ce résultat statistique du traitement des demandes d'asile, résultat tributaire des choix de politique publique ${ }^{29}$. Trois chercheurs suisses ont également illustré cette dépendance en comparant le traitement de 180000 demandes d'asile dans les vingt six cantons suisses entre 1988 et 1996 : ils expliquent la variabilité des taux de rejet d'un canton à l'autre et d'une nationalité à l'autre en fonction du canton, notamment par les modalités d'organisation des services traitant les dossiers ${ }^{30}$.

18 Cette corrélation des taux de rejet et des choix de politique publique apparaît aussi dans une perspective historique. En France, dans les décennies cinquante et soixante, 
jusqu'au milieu des années 1970, la très grande majorité des récits de demande d'asile étaient considérés comme crédibles $^{31}$. La procédure d'octroi est alors expéditive. Elle le restera. Depuis lors, la très grande majorité des récits ne sont plus crus. On passe en quelques années d'une dynamique d'octroi expéditif au bénéfice d'un petit nombre à une dynamique de rejet expéditif au détriment du plus grand nombre : hormis quelques populations privilégiées d'exilés pour lesquels sont instaurés des quotas de protection (chiliens, cambodgiens, vietnamiens, laotiens) les demandes d'asile de toutes les autres origines, politiquement non désirées, vont être administrativement considérées comme non crédibles. Il faut environ dix ans pour que l'ensemble des agents publics, fonctionnaires et juges, s'adapte mentalement à cette norme politique. Durant le temps de cette adaptation, assurée aussi par les politiques de recrutement des agents publics, le taux de rejet global augmente inexorablement. Il augmente au fur et à mesure où diminue la part des demandes issues de nationalités privilégiées par le gouvernement. Il augmente corrélativement quand s'accroît la part des demandes d'asile politiquement et diplomatiquement non désirées notamment celles d'origine africaine dont le taux de rejet est d'environ 90\%, depuis le milieu des années 1980 jusqu'à aujourd'hui.

Le passage de l'octroi expéditif au rejet expéditif n'a pas lieu au même moment dans tous les pays, mais leurs taux de rejet convergent tous vers le même sommet. Le mouvement d'ensemble apparaît bien dans le cadre chronologique allant de 1985 à 1995 :

\begin{tabular}{|l|l|l|l|l|l|l|l|}
\hline & Autriche & Belgique & France & Allemagne & Italie & Suisse & Royaume-Uni \\
\hline 1985 & 55 & -- & 57 & 60 & 93 & 86 & 78 \\
\hline 1986 & 64 & -- & 61 & 78 & 96 & 88 & 88 \\
\hline 1987 & 69 & -- & 67 & 88 & 93 & 91 & 87 \\
\hline 1988 & 73 & 62 & 65 & 89 & 96 & 93 & 75 \\
\hline 1989 & 81 & 51 & 72 & 94 & 64 & 95 & 67 \\
\hline 1990 & 93 & 63 & 85 & 95 & 40 & 95 & 74 \\
\hline 1991 & 87 & 74 & 80 & 92 & 95 & 97 & 91 \\
\hline 1992 & 90 & 72 & 72 & 95 & 95 & 95 & 97 \\
\hline 1993 & 92 & 71 & 72 & 95 & 91 & 85 & 92 \\
\hline 1994 & 92 & 69 & 76 & 90 & 82 & 87 & 95 \\
\hline 1995 & 87 & 68 & 84 & 83 & 83 & 85 & 94 \\
\hline
\end{tabular}


21 Certains pays comme l'Italie, la Suisse et le Royaume-Uni plafonnaient déjà depuis le milieu des années 1980, d'autres comme l'Autriche, la France et l'Allemagne évoluent encore dans les années 1990 tandis que les derniers comme la Belgique ou les nouveaux pays d'immigration (Espagne, Grèce...) les rejoignent à la fin de cette décennie. Ces chiffres ne tiennent pas compte des formes de protection dites "subsidiaires»: apparues récemment elles ne sont pas pertinentes dans des séries statistiques de longue période et s'agissant de protection de courte durée elles peuvent s'analyser comme des rejets différés dans le temps.

Il reste encore aujourd'hui quelques disparités entre pays européens mais elles tendent à se réduire au fur et à mesure qu'ils convergent vers un plafond commun. Les derniers chiffres disponibles sont parlants :

\begin{tabular}{|l|l|l|l|}
\hline Allemagne* & 95.7 et 85.7 & Irlande & 94.1 et 82.3 \\
\hline Autriche & 70.4 & Italie* & 88.7 \\
\hline Belgique & 76.1 et 90.1 & Luxembourg & 93.1 \\
\hline \hline Danemark* & 75.5 et 92.8 & Pays-Bas* & 98 et 92.3 \\
\hline Espagne* & 81.4 & Portugal* & 90.9 \\
\hline Finlande* & 99.5 & Royaume-Uni* & 96.7 et 79.6 \\
\hline France & 90.2 et 88.6 & Suède* & 98.3 et 98.5 \\
\hline \hline Grèce & 99.9 & & \\
\hline
\end{tabular}

Tableau n ${ }^{\circ} 2$ : Taux de rejet des demandes d'asile conventionnel

( $1^{\text {ère }}$ instance et appel) en $2003^{33}$

Certains pays ont parcouru le chemin de cette évolution jusqu'à sa limite ultime, les autres s'en approchent. Quelle est cette limite? L'exemple grec montre qu'il n'y en a qu'une qui soit absolue : celle des $100 \%$. Dans d'autres pays, où la procédure d'asile a généré progressivement des budgets annuels et un volume d'emplois publics conséquents, il existe des freins bureaucratiques à cette évolution. La proximité des $100 \%$ fait notamment courir le risque d'une remise en question de ces budgets et emplois puisqu'un tel résultat, proche ou égal à $100 \%$, pourrait facilement être obtenu à un coût budgétaire proche ou égal à zéro.

\section{Les usages politiques des taux de rejet}

Dans les institutions administratives, juridictionnelles et associa-tives de l'asile, les exilés dont la demande d'asile a été rejetée ne sont habituellement pas appelés « fauxréfugiés » mais « déboutés du droit d'asile ». La pudeur technocratique a ses raisons : le débouté n'est pas un objet de haine. Certains agents publics (administrateurs, juges...), ou quasi publics (salariés d'associations financées par l'Etat...), peuvent être exaspérés par les «mensonges». Mais un mensonge éventé donne au censeur un sentiment flatteur de lucidité et peut donc l'incliner aussi à une indulgente indifférence. Pour 
d'autres agents, le débouté disparait simplement dans la foule anonyme des monceaux de dossiers rejetés avec quelques fois le sentiment amer d'une possible « erreur » dont la portée exacte est difficile à connaître. Le "faux-réfugié ", tel qu'il apparaît dans l'espace public est, comme on va le voir, différent du " débouté ». C'est une menace, un sujet d'inquiétude ; fraudeur présentant un récit contrefait de persécutions et abusant du droit d'asile; parasite souhaitant vivre des miettes de prospérité enlevées au pays d'accueil ; envahisseur du fait du nombre de demandes d'asile et surtout du gisement insondable dont elles sont issues.

Distinguer abstraitement le «débouté » administratif et le «faux-réfugié » plus politique, ne signifie pas, cependant, qu'ils soient dissociés dans la réalité sociale. Dans la plupart des pays européens, les taux de rejet s'élèvent durant les années 1980 c'est-àdire généralement avant que l'imagerie du faux-réfugié n'envahisse les discours politiques et les mass-medias. Et cette donnée statistique distillée avec constance, quels que soient les évènements dans le reste du monde, depuis des décennies, a façonné en profondeur les croyances qui dominent aujourd'hui la vie politique au sujet des « fauxréfugiés ».

Certains acteurs politiques ont une connaissance, il est vrai, quelque peu approximative de ces statistiques. Le Premier ministre britannique, dans sa proposition d'externalisation de l'asile, sous estime ainsi la moyenne européenne : "La moitié ou les trois quarts de ceux qui demandent l'asile en Europe ne remplissent pas les critères pour être considérés comme pleinement réfugiés $»^{34}$. Son principal opposant, Ducan Smith (leader du Parti Conservateur), plus prudent dans l'estimation du chiffre, va plus loin dans l'analyse : « La grande majorité de ceux qui arrivent, vient pour des raisons qui n'ont rien à voir avec de véritables persécutions politiques. Ils viennent pour des raisons économiques ou, pour un nombre plus petit mais déjà significatif, pour des raisons criminelles ou terroristes $»^{35}$.

Lors de la dernière réforme du droit d'asile en France, le ministre des Affaires Etrangères s'exprimant devant l'Assemblée Nationale est plus précis :

«Certes, l'afflux de demandeurs d'asile témoigne de l'aggravation des violations des droits de l'Homme et des persécutions à l'échelle de la planète. Chaque jour, des hommes et des femmes n'ont d'autre solution que de fuir leur pays pour échapper à des traitements dégradants, à la torture, à la mort. Mais les personnes réellement persécutées sont loin de représenter la majorité des demandeurs d'asile : alors qu'il reconnaissait le statut de réfugié à près d'un demandeur sur cinq il y a peu, l'OFPRA ne l'accorde plus aujourd'hui qu'à moins de $13 \%$ des demandeurs. Le constat est encore plus net quant à l'asile territorial, pour lequel le taux de décisions favorables n'a pas dépassé $0,3 \%$ en 2002. Le fait est que beaucoup d'étrangers sollicitent notre système d'asile, non pas pour obtenir la protection de notre pays, mais pour s'y maintenir le plus longtemps possible, leur motivation étant de nature économique $\Perp^{36}$.

Dans cette vision du monde, quelques réfugiés authentiques s'entremêlent au flot des migrants économiques. Le phénomène a été conceptualisé très tôt dans le langage technocratique international par la notion de «flux mixtes». Permettant de rappeler cette réalité bien connue de manière sobre et pudique, elle est devenue d'usage courant au Haut-Commissariat aux Réfugiés de l'ONU ou à la Commission européenne :

«Alors que les admissions au statut de réfugié défini par la Convention de Genève connaissaient au début de la décennie 1990 une certaine stabilité en valeur absolue en dépit des variations de la demande, on constate au cours des dernières années un relatif déclin. Il pourrait s'expliquer par les raisons suivantes :

- des mesures adoptées par des Etats membres ou par l'Union européenne qui 
auraient pour effet de détourner certains flux de réfugiés vers d'autres destinations ou de dissuader certains réfugiés de formuler une demande d'asile ;

- l'hypothèse avancée par certains de flux de plus en plus mixtes où une migration économique serait dissimulée sous les demandes d'asile, ce qui entraîne naturellement des rejets des demandes;

- une inadéquation croissante entre la nature de la demande et les critères de la Convention de Genève. Ceci s'explique en grande partie par la recrudescence de conflits armés entraînant une situation d'insécurité généralisée et des violations des droits de l'Homme qu'il est difficile de faire coïncider avec la notion de persécution telle que l'appréhende une longue tradition d'interprétation de la Convention de Genève en Europe ${ }^{37}$.

D'autres causes sont fréquemment invoquées pour expliquer cette mixité croissante des flux, notamment l'action de mafias gestionnaires des filières de clandestins. Ainsi le Comité des affaires intérieures de la Chambre des Communes présente la courbe historique du nombre de rejets de demandes d'asile avec ce commentaire : «Il y a de bonnes raisons de penser que cette croissance considérable en nombre au cours de la dernière décennie indique non pas une croissance des réfugiés authentiques (genuine refugee) mais une croissance des abus du système de l'asile par des migrants économiques qui sont dans bien des cas exploités par des gangs criminels hautement organisés $»^{38}$.

L'évocation de cette criminalité organisée dont les exilés seraient victimes permet de ne pas leur laisser l'entière responsabilité du problème migratoire. Elle permettra aussi ultérieurement de justifier avec humanité leur reconduite dans leur pays d'origine. Car l'ensemble des positions exprimées dans ce débat revendique un attachement aux valeurs humanistes, y compris le droit d'asile. C'est même au nom de sa défense que sont stigmatisés les faux-réfugiés par Raymond Hall, responsable du Bureau Europe du $\mathrm{HCR}$, qui appelle à «débarrasser les procédures nationales de l'asile de ces abus particulièrement exaspérants qui constituent l'un des facteurs de remise en cause actuelle de la crédibilité des procédures nationales de l'asile ${ }^{39}$. Le même souci de défense du droit d'asile est exprimé par le ministre de l'Intérieur britannique s'affligeant des torts qui lui sont causés par les abus ${ }^{40}$.

Or l'ensemble des explications - flux mixtes, mafias de passeur... - et des implications menaces sur le droit d'asile, sur les valeurs humanistes... - est toujours fondé et démontré par les taux de rejet, comme le rappelle la Commission européenne dans sa communication de novembre 2000 :

«La crise du système d'asile est de plus en plus évidente dans un certain nombre d'Etats membres et un malaise grandissant est ressenti par l'opinion publique. On observe une utilisation abusive des procédures d'asile et un gonflement des flux mixtes, souvent entretenus par le trafic et la traite des êtres humains et composés à la fois de personnes ayant légitimement besoin d'une protection internationale et de migrants utilisant les voies et les procédures d'asile pour accéder au territoire des Etats membres, à la recherche d'une amélioration de leur situation économique. Ainsi les taux de décisions négatives après examen de tous les besoins de protection internationale demeurent importants. Ce phénomène, qui constitue une menace réelle pour l'institution de l'asile et plus généralement pour la tradition humanitaire européenne, requiert des réponses structurelles» ${ }^{41}$.

Le problème de l'envahissement des faux-réfugiés a pris une ampleur considérable puisque c'est l'ensemble des valeurs fondatrices de la culture européenne qui est en cause en plus des intérêts financiers. Des réponses structurelles verront effectivement le jour avec les politiques actuelles de bannissement et d'enfermement. 


\section{La radicalisation des rhétoriques de rejet} d'automaticité dans l'octroi du statut de réfugié. Elle définit des critères qui subordonnent implicitement cette attribution à une évaluation du récit d'exil. Or les conditions de procédures et de dotations administratives déjà vues, ne permettent pas aux agents de répondre à la question : s'agit-il d'un vrai réfugié ? Aussi répondent-ils à la seule question traitable : est-ce que cet exilé m'a convaincu? Et l'inclination à croire ou ne pas croire dépend de facteurs sociaux et psychologiques pour beaucoup sans lien avec le récit évalué (convictions politiques de l'examinateur notamment en matière d'immigration, connaissances sur le pays d'origine, compréhension des conditions sociales d'expression de la requête, interprétation personnelle d'une Convention imprécise aux jurisprudences chaotiques, etc.). Le sentiment individuel d'objectivité et de justice, auquel peut aspirer l'évaluateur dans chaque décision administrative ou politique, devient de ce fait fortement tributaire des idéologies politiques de l'époque. Et il est alors, pour tous, plus difficile de croire les exilés que de s'en défier. La rhétorique de l'incrédulité s'énonce simplement : je n'y crois pas. Elle est difficilement discutable.

La rhétorique de l'exclusion - Elle se forme aussi dans la Convention de Genève sur les réfugiés qui prévoit d'exclure tyrans, bourreaux et droits communs fuyant la justice de leurs pays. Cette rhétorique visait à renforcer la protection des victimes en écartant les auteurs de persécutions (qui conservèrent toujours, il est vrai, le bénéfice du pouvoir discrétionnaire de protection par les Etats amis). Dans la procédure d'asile ces exclusions sont rares... autant que les aveux spontanés de culpabilité en l'absence desquels il est bien difficile de juger sans investigation, sur de simples soupçons, des faits parfois terribles (génocides, crimes de guerre...). Cependant la rareté n'est qu'apparente puisque rien ne fait obstacle à des exclusions implicites; un simple soupçon, précisément, de terrorisme par exemple, peut décrédibiliser une demande 
d'asile et toutes celles de la même origine. Dans la pratique administrative et juridictionnelle, la rhétorique reste officiellement celle de l'incrédulité, économisant l'exposé d'autres motifs de rejet qui demeurent ainsi implicites. Cette pratique sousjacente à bon nombre de décisions s'accroît sous l'effet des discours et politiques sécuritaires qui affectent partout en Europe les demandes d'asile ${ }^{42}$; certaines demandes d'asile plus que d'autres faut-il ajouter : en particulier celles qui sont exprimées par des arabes, des kurdes, des tchétchènes, des irakiens, des tamouls...

La rhétorique du déni d'asile - La notion de demande « manifestement infondée » apparaît dès $1980^{43}$ dans le langage technocratique pour désigner le moyen procédural de rejeter plus vite encore que d'habitude, sans audition, voire à lecture rapide du récit d'exil. Le HCR conceptualise en $1983^{44}$ cette pratique de rejet déjà bien établie par les Etats dès la fin des années 1970. Le refus d'entendre une demande ou de permettre qu'elle s'exprime pleinement s'institutionnalise ensuite aux postes frontières, dans les aéroports, aux guichets administratifs et dans les procédures juridictionnelles. Ils gonflent les taux de rejet dans des proportions variables selon les pays : le RoyaumeUni les estime aujourd'hui officiellement à près de $40 \%$. La notion s'articule ensuite avec celle de "pays sûr ", aux multiples déclinaisons - "pays d'origine sûr ", «paystiers sûr " (par exemple de transit), " pays de premier accueil » sûr, ... - conceptualisée par le HCR en $1989^{45}$. La publicité des listes de pays sûrs posant des problèmes diplomatiques (pour ceux qui n'y sont pas!), elles demeurent souvent officieuses mais facilitent aussi bien et sans plus d'égard les rejets des demandes d'asile concernées ainsi considérées a priori comme " manifestement infondées».

40 La rhétorique de l'externalisation - Elle ne constitue pas une innovation radicale mais s'inscrit dans le prolongement de ce qui précède comme le dernier avatar en date d'un mouvement de radicalisation. Concept descriptif et non juridique, son histoire reste à entreprendre. Sur le plan rhétorique elle s'amorce à la fin de la décennie 1990 avec la notion «d'asile interne ». Elle signifie en substance qu'une personne pouvant trouver refuge dans une autre partie de sa patrie doit être regardée comme infondée à le chercher dans un autre pays. La même logique inspire le concept de «zones de protection spéciale » élaboré par le HCR afin de concentrer les réfugiés d'une région, toutes origines confondues, dans un lieu de regroupement forcé. L'apport britannique semble se limiter aux "camps de transit pour le traitement des demandes d'asile" permettant de rejeter les demandes avant l'entrée sur le territoire et de transférer à d'autres Etats la gestion des camps et de l'expulsion. Plus récemment le concept italoallemand de "portails de l'immigration" vise dans la même logique à organiser le «tri » des exilés avant leur accès au territoire européen. Les délibérations politiques relatives à ces projets très actuels seront présentées dans la troisième partie de cet article.

\section{Le tournant national-sécuritaire : l'exilé comme menace}

41 Si on considère le système sociologique que forment ensemble les ministres et hauts fonctionnaires du secteur, les administrateurs et juges de l'asile, la spirale du rejet s'analyse d'abord comme un phénomène endogène. Celui-ci prend cependant une portée nouvelle quand s'opère un autre mouvement de plus large ampleur, d'abord xénophobe puis sécuritaire. 

succès électoraux de l'extrême droite. Pourtant ni cette notion, ni ces succès ne suffisent à expliquer l'évolution des politiques de l'immigration et de l'asile. Les études disponibles sur l'Autriche ${ }^{47}$, l'Espagne ${ }^{48}$, la France ${ }^{49}$, la Grèce ${ }^{50}$, l'Italie ${ }^{51}$ et le Royaume$\mathrm{Uni}^{52}$ montrent que ce tournant, que l'on peut qualifier de national et également sécuritaire, met en jeu des élites dirigeantes: ministres, conseillers, hautsfonctionnaires, autres agents publics et semi-publics, experts, lobbyistes, chargés de communication... Tous n'ont pas besoin, loin s'en faut, de tenir compte d'une opinion publique souvent enrôlée à peu de frais dans la justification des positions politiques de ceux qui s'expriment.

Pour le montrer, encore faut-il ne pas réserver l'exclusivité du fait xénophobe à quelques groupuscules ou partis ascendants, ne pas en exonérer a priori les élites dirigeantes, administratives et politiques. On peut alors distinguer et comparer xénophobie contestataire (celle des partis d'extrême droite) et xénophobie gouvernante (celle des politiques anti-migratoires) et montrer l'antériorité de celle-ci. Aucune des deux ne laisse l'exilé indemne. Il devient une menace, un ennemi intérieur et extérieur, qui donne lieu à criminalisation et à des incarcérations croissantes. Face aux montées en puissance des idées xénophobes et sécuritaires d'autres idées sont en recul et leurs soutiens en déclin. Cette raréfaction des soutiens à la cause des exilés, moins étudiée, nécessite encore des recherches complémentaires ${ }^{53}$.

\section{Xénophobie contestataire et xénophobie de gouvernement}

En 1999, les partis d'extrême droite étaient représentés aux parlements nationaux en Autriche, Belgique, Danemark, Italie, Norvège, Suisse et, au niveau local, en France et, dans une moindre mesure, en Allemagne (les Pays-Bas viennent s'ajouter à cette liste en 2002). Dans quatre pays (Autriche, Italie, Pays-Bas et Suisse) ils rejoignent au moins une fois, depuis lors, une coalition gouvernementale. Ce phénomène semble donner une explication simple et évidente à l'évolution des politiques de l'asile et de l'immigration. Une approche historique et comparative fragilise pourtant une telle interprétation. En la retenant de manière exclusive, on oublierait que les lois et discours ministériels, avec toute l'autorité symbolique qui est la leur, mais aussi les actes administratifs à tous les échelons de la hiérarchie, diffusent des idées, des croyances et des représentations sociales avec autant d'efficacité sinon plus que les partis politiques.

En France, les éléments fondamentaux des politiques anti-migratoires sont posés dès les années 1970, près de dix ans avant l'émergence électorale du Front National (FN) en 1983 : fermeture des frontières (1974), restrictions au regroupement familial (1976), pénalisation du séjour irrégulier (1980), contrôles d'identités au faciès (1981). Comme l'observe Danièle Lochak ${ }^{54}$ l'alternance de 1981 n'ouvre qu'une brève parenthèse associant des mesures spectaculaires en faveur des sans-papiers, et les prémices, dès 1982, de la conversion socialiste à la lutte anti-migratoire: durcissement contre les irréguliers, mise en opposition des anciens et nouveaux migrants. La victoire électorale du FN en mars 1983 marque les esprits mais il ne s'agit encore que de scores réduits et rien n'indique alors qu'ils seront durables. Pourtant, dès l'année suivante le Premier ministre socialiste L. Fabius s'adapte: "l'extrême droite, ce sont de fausses réponses à de vraies questions ». En 1986, le retour de la droite au gouvernement, accompagné par l'entrée du FN à l'Assemblée Nationale, donne lieu à des surenchères gouvernementales 
et partisanes dans la répression des migrants : les charters d'expulsés deviennent un instrument de communication politique du ministre de l'Intérieur C. Pasqua et le jeu politique se recompose autour de ce nouvel acteur qu'est le FN. L'évolution affecte tous les esprits: "On ne peut pas accueillir toute la misère du monde " résume le Premier ministre M. Rocard en 1989. Les discours et les actes publics se sont modifiés en France bien avant que l'extrême droite n'affecte significativement le jeu électoral. La première grande loi italienne sur l'immigration, dite «loi Martelli » (héritier de Bettino Craxi), date de 1986. Elle fait émerger l'immigration comme problème public dans la vie politique italienne. Bien que libérale par certains aspects, elle introduit les premiers dispositifs restrictifs et stigmatisants à l'égard des exilés. A côté de mesures favorables au marché du travail (demandes de régularisation par les employeurs, centres d'accueils et d'orientation, facilités pour certaines nationalités demandées...), la loi autorise les expulsions d'exilés sans papiers, incluant explicitement et contre le droit international les demandeurs d'asile et les étrangers accusés de crimes ${ }^{55}$. En 1993, le ministre de la Justice Giovanni Conso simplifie les procédures d'expulsion d'exilés placés en préventive ou gardés à vue pour dommages à la propriété, vol et recels; il multiplie également les motifs d'incarcération et d'expulsion. En 1995 le « décret Dini » crée des sanctions nouvelles contre ceux qui emploient et transportent des exilés sans papier $^{56}$. Or l'émergence électorale de la Ligue du Nord date de $1987(20 \%)$ et se limite à la Lombardie. A cette date, la " loi Martelli » marque le débat politique depuis déjà un an. La Ligue qui s'organise nationalement en 1991 et apparaît électoralement dès 1992 n'obtient qu'en 1996 un score $(10,1 \%)$ qui lui permet d'imposer des négociations de coalition aux partis de gouvernement. Cela fait déjà plusieurs années que les politiques publiques menées par les gouvernements ont endossé les idées de la lutte contre les exilés.

Jusque dans les années 1980 l'Autriche revendique une tradition d'accueil des réfugiés (germaniques mais aussi hongrois, tchèques, slovaques et polonais après la guerre). Ceux de l'Est furent longtemps perçus comme des héros échappés de la tyrannie communiste. A partir de 1989 s'opère une dégradation brutale des discours politiques à leur sujet. Dès le milieu des années 1990, observe Ruth Wodak, la tolérance paternaliste dont bénéficiaient les réfugiés de l'Est se transforme en hostilité à l'égard des mêmes, devenus "migrants économiques » trop paresseux et égoïstes pour demeurer chez eux et $\mathrm{y}$ résoudre leurs propres problèmes ${ }^{57}$. Les politiques publiques se durcissent; tant celles des conservateurs que des sociaux-démocrates: diminution draconienne du quota d'immigration annuelle, restrictions dans le renouvellement des cartes de séjour, expulsions musclées des étrangers sans papiers... ${ }^{58}$ 'administration s'exprime aussi, à sa manière: les courriers de notification des refus de regroupement familial, par exemple, multiplient et banalisent les arguments xénophobes; les services concernés ne trouvent pas motifs à remplir plus de $63 \%$ des quotas annuels pourtant officiellement ouverts aux demandes de regroupement familial ${ }^{59}$. L'ensemble du secteur des politiques de l'immigration et de l'asile glisse vers la xénophobie dès les années 1990. Et, même si les premiers succès du Freiheitliche Partei Österreich de Jörg Haider datent de 1986 (9,7\% aux élections locales) et que la décennie 1990 est marquée par des accords locaux de gouvernement entre le FPÖ et le principal parti conservateur (l'ÖVP de Wolfgang Schüssel), l'explosion du FPÖ au niveau national date de 1999 (27\%) et le premier accord national de gouvernement de février 2000. 
L'extrême droite ne perturbe la vie politique britannique qu'à partir de $2001^{60}$ et la perturbation reste très limitée ${ }^{61}$. En outre, jusqu'en 1999, le nombre annuel de demandes d'asile reste inférieur à $50000^{62}$. Pourtant, dès le début des années 1990, observe Ron Kaye ${ }^{63}$, les partis politiques façonnent un consensus contre les demandeurs d'asile par un langage disqualifiant. Les mass-medias amplifient cet usage politique des termes « fraudes », « parasites », « tricheries » et " réfugiés économiques » au sujet des demandeurs d'asiles ${ }^{64}$. Dès cette époque sont opposés « réfugiés authentiques » (genuine refugees) sujets à compassion et "faux-réfugiés" (bogus refugees) sans droits, ni défense $^{65}$. Face à l'augmentation de leur nombre (de faible ampleur comparée aux autres chiffres européens et liée à la guerre en Yougoslavie), les conservateurs réagissent en 1993 par des législations restrictives et un langage d'hostilité. Les travaillistes sont divisés : les vieux députés de base du Labour dénoncent le racisme des législations de 1993 et 1996. Mais les figures montantes du New Labour comme Tony Blair disent comprendre le "problème", la nécessité de lutter contre les "abus du droit d'asile »; et, de fait, leur arrivée au pouvoir en 1997 n'infléchit pas la tendance. La loi de 1999 sur l'immigration et l'asile affiche une hostilité grandissante à l'égard des exilés : bons d'approvisionnement stigmatisant, dispersions forcées hors de Londres, accroissement des incarcérations... Or en 1999, le British National Party n'a aucun poids électoral, aucune surface sociale : il est présenté dans la presse comme un groupuscule, son nombre d'adhérents est estimé à 1500 dont moins de 200 assistent aux meetings ${ }^{66}$.

\section{Des logiques sécuritaires à la réclusion des exilés}

Ce tournant nationaliste et xénophobe intervient dans un environnement international bouleversé à partir des années 1990. Comme l'observe Malcolm Anderson, "après l'euphorie collective qui suivit la chute du Mur de Berlin, des craintes apparurent, en partie véhiculées par les gouvernements et les appareils d'Etat ${ }^{67}$. La menace directe contre l'Europe ayant disparu, l'ensemble du schéma de sécurité collective devint matière à réflexion et l'on s'intéressa à de nouvelles menaces. La première menace envisagée fut celle d'un déferlement des réfugiés en provenance de l'Est. Il n'eut pas lieu. Mais ce démenti par la réalité ne freina pas la diffusion de cette théorie politique d'un risque de déferlement migratoire. Cette nouvelle menace fut construite par association de phénomènes souvent indépendants : trafics de stupéfiants, réseaux terroristes, filières de prostitutions, migrations économiques, fondamentalisme musulman, etc. «En liaison avec ces questions posées par les pouvoirs politiques, [note Anderson en 1997], une dimension d'anxiété culturelle investit la politique des sociétés européennes». Avec les questions viennent très vite les réponses apportées par les mêmes; ou, plus exactement, lorsque les réponses sont déjà là, il reste à construire les problèmes, notamment les menaces, justifiant l'emploi des solutions politiquement préférées. Sécurité et immigration font alors bon ménage dans la reconversion des référentiels de l'action policière, douanière, diplomatique mais aussi sociale ou éducative tendant vers ce que Didier Bigo nomma une "gouvernementalité par l'inquiétude": "Le discours sécurisant l'immigration est alors en position de force symbolique et devient une technologie politique, une modalité de la gouvernementalité contemporaine ${ }^{68}$.

50 Dans cette perspective, l'enfermement des migrants devient un élément crucial des politiques anti-migratoires avec deux effets latéraux importants: il renforce la dangerosité socialement perçue des migrants tout en affichant la mobilisation des autorités contre cette menace. De nombreuses études de sciences sociales disponibles 
sur cette forte fréquence d'emprisonnement des étrangers en Europe aboutissent, observe Anastassia Tsoukala ${ }^{69}$, à des conclusions convergentes : ces effectifs d'étrangers en prison sont tirés à partir des années 1980 par les infractions aux législations sur l'immigration (séjour irrégulier, refus d'expulsion.... $)^{70}$, par les infractions liées directement à la vie en clandestinité (faux et usages de faux, infraction à la législation sur l'emploi...) et par les incarcérations préventives d'autant plus nombreuses que les étrangers ne présentent pas les garanties requises (stabilité et légalité du séjour, du domicile, de la situation familiale, de l'emploi, de la scolarité, etc.) pour bénéficier des mesures alternatives à la détention préventive. Reste, au-delà de ces facteurs, une surreprésentation des étrangers liée non à leurs origines nationales mais à leurs distributions statistiques suivant d'autres variables: l'âge, le sexe et les conditions socio-économiques. Si l'on efface, au moyen de calculs statistiques, l'influence de ces variables pour mettre en évidence l'effet propre à la variable "nationale », comme cela été fait pour l'Allemagne ${ }^{71}$, on s'aperçoit alors que la population étrangère n'est pas plus criminogène que la population nationale. L'essentiel réside donc dans cette corrélation entre les taux d'incarcération d'étrangers et les facteurs liés au séjour irrégulier, comme le montre l'étude réalisée par James Linch et Rita Simon en $1998^{72}$ sur sept pays (USA, Canada, Australie, Royaume-Uni, France, Allemagne, Japon). A cette corrélation fondamentale s'ajoutent des facteurs incidents notamment les discriminations policières et judiciaires. L'ensemble de ces analyses permet donc de considérer les taux d'incarcération des étrangers comme une mesure sociologiquement pertinente du degré de criminalisation de l'exilé dans une société :

\begin{tabular}{|l|l|l|l|l|}
\hline & 1983 & 1988 & 1991 & 1997 \\
\hline Autriche & 7 & 9 & 22 & 27 \\
\hline Belgique & 22 & 30 & 34 & 38 \\
\hline France & 25 & 26 & 30 & 26 \\
\hline Allemagne & -- & 15 & 15 & 34 \\
\hline Grèce & 12 & 19 & 22 & 39 \\
\hline Italie & 8 & 9 & 15 & 22 \\
\hline Pays-Bas & 23 & 20 & 25 & 32 \\
\hline Espagne & 8 & 12 & 16 & 18 \\
\hline Suède & 17 & 22 & 20 & 26 \\
\hline Suisse & 32 & 40 & 44 & - \\
\hline
\end{tabular}

Tableau n ${ }^{\circ} 3$ : Proportion d'étrangers dans les prisons européennes (en \%) ${ }^{73}$

Ce phénomène de criminalisation est continu. Les sommets de son palmarès en 2000 sont édifiants : $62,6 \%$ en Suisse, $48,4 \%$ en Grèce, $40,4 \%$ en Belgique, $34,1 \%$ en Allemagne, 
$30,1 \%$ en Autriche... Pour être plus précis, il faut rapporter la proportion d'étrangers dans les prisons à ce qu'ils représentent dans la population nationale. En adoptant cette méthode Dario Melossy ${ }^{74}$ produit des ratios qui séparent nettement deux groupes de pays : au sud de l'Europe on trouve les plus fortes disproportions d'étrangers dans les prisons (Grèce : 19,4 - Espagne : 18,8 - Italie : 15 - Portugal : 13,5); les autres pays ont quand même entre deux et sept fois plus d'étrangers dans leurs prisons que ce que ces étrangers représentent dans la population (la Belgique se singularise par un ratio 10 intermédiaire). Cette situation au sud de l'Europe a plusieurs explications: la criminalisation des migrants y est forte par suite d'une réaction brutale face à un phénomène inhabituel dans des pays qui, jusqu'au milieu des années 1980, sont des pays d'émigration et découvrent alors l'immigration. Surtout, ces législations antimigratoires sont progressivement adoptées sous la pression de l'Union européenne et de ses autres Etats membres qui considèrent ces pays du sud comme les gardiens laxistes d'une frontière commune au sud de l'Europe. Cette pression s'accentue au milieu des années 1990 avec le traité Schengen qui mutualise les frontières extérieures et surtout les débats politiques relatifs à leur contrôle. Elle fut marquée à l'égard de tous ces pays, mais particulièrement à l'égard de la Grèce dans un premier temps exclue de l'espace Schengen : elle dut s'adapter pour l'intégrer et se trouve aujourd'hui avec le plus fort taux de rejet des demandes d'asile (99,9\% en 2003), le plus fort taux d'incarcération d'étrangers (48,4\% en 2000) et la plus forte disproportion d'étrangers emprisonnés (19,4 fois plus que de nationaux). Comme le montre une étude de 2001 sur cette tendance en Grèce, les incarcérations sont dues massivement à des peines liées aux séjours irréguliers, à des détentions préventives spécifiques aux étrangers et à l'emprisonnement de ceux qui ne peuvent pas être expulsés ${ }^{75}$.

Les événements du 11 septembre 2001 vont entraîner une accélération considérable du phénomène de criminalisation des exilés. A cet égard, toutes les études sont convergentes. Mais cette actualité éblouissante risque aujourd'hui de nous empêcher de percevoir la profondeur historique du phénomène et donc d'en mesurer correctement l'ampleur. Toutes les tendances précédemment décrites sont antérieures à 2001 .

\section{Le déclin des soutiens aux exilés}

Les politiques de l'immigration et de l'asile peuvent être interprétées comme le reflet d'une concurrence entre deux coalitions : la coalition nationale et sécuritaire, en se renforçant, entraîne le tournant précédemment décrit; l'autre coalition, celle de la défense des exilés et de l'asile va en déclinant. Or ce déclin demeure peu étudié alors qu'il intervient dans la modification du rapport de force entre les deux coalitions. Une illustration en est donnée par Monica den Boer sur le débat relatif au coût des exilés : "L'absence d'un effort coordonné pour miner l'opinion largement répandue, qui fait de l'immigrant un concurrent redoutable dans la redistribution, étonnamment réduite, de l'emploi et du Welfare, fait partie de cette stratégie de sécurisation: à cet égard le silence est aussi une parole $\aleph^{76}$. L'ampleur du tournant national-sécuritaire tient, en partie au moins, à l'affaiblissement des discours et des actions allant dans un sens opposé ainsi qu'à des repositionnements et reclassements politiques de certains acteurs. Considéré à l'échelle de l'Europe, ce déclin des soutiens aux exilés peut être observé tant au niveau des acteurs nationaux que de ceux qui interviennent dans l'européanisation de l'action publique. 
55 Sur le plan national, il est difficile d'avoir une vue panoramique de ce qui se passe en Europe du fait de la rareté des études. Néanmoins, les observations que l'on peut faire sur la France, pays d'immigration ancienne, et sur la Grèce, pays d'immigration nouvelle, font ressortir des phénomènes susceptibles d'affecter d'autres pays et allant clairement dans le sens d'un affaiblissement des soutiens aux exilés.

56 Le cas de la France ${ }^{77}$ illustre parfaitement le phénomène de désengagement dont trois formes peuvent être présentées, sans prétendre les ordonner ou les hiérarchiser. Premièrement, les partis de gauche qui dans les années 1970 affichaient tous leur engagement en faveur des exilés, disparaissent de ce combat en accédant au pouvoir rompant au passage les " courroies de transmission » avec les associations. La dernière réforme de l'asile, en 2003, permet un état des lieux: les consignes de la direction socialiste à ses troupes parlementaires sont explicitement celles d'une non mobilisation; la direction communiste s'exprime par son silence; certains Verts s'engagent tandis que d'autres se taisent. Ensuite, une partie des associations spécialisées $^{78}$ dans l'aide aux exilés connaissent au cours des années 1990 un mouvement rapide de "professionnalisation ». Le nombre de leurs salariés augmente au fur et à mesure de leurs engagements dans la gestion de services financés par les pouvoirs publics d'accueil et d'hébergement d'exilés. Cette privatisation de services sociaux transforme ces associations originellement militantes en structures paraadministratives. Mais elles restent sur la scène médiatique, toujours sollicitées par les journalistes au titre de la "société civile» malgré leur changement d'identité sociologique et la subordination politique qui en résulte vis-à-vis des autorités. Enfin, une autre catégorie d'association fait défaut: celle des associations issues de l'immigration (comme des associations d'algériens, de marocains, de mauritaniens, etc.). Plus puissantes que les précédentes par le nombre de leurs adhérents et leurs fonds propres, elles représentent des migrants déjà installés et sont souvent tributaires de la diplomatie des Etats d'origine. Elles se mobilisent essentiellement sur les thèmes de l'intégration, de la multiculturalité et de la non-discrimination mais délaissent tendanciellement la cause des nouveaux migrants (droit d'asile, mouvements de sanspapiers...). Elles sont d'ailleurs incitées à ces choix par les discours gouvernementaux de droite et de gauche opposant les migrants anciens à intégrer et les migrants nouveaux à réprimer.

57 Le cas de la Grèce illustre non pas un processus de désengagement mais la quasiinexistence d'un milieu militant en faveur des exilés. Ce phénomène assez général dans ce pays de faible militance associative est particulièrement marqué au sujet de l'immigration que la Grèce découvre récemment, dans les années $1990^{79}$. Les associations issues de l'immigration sont récentes : la première fédération (Forum Grec des Migrants) date de 2001 et les moyens de ses organisations membres se limitent souvent à une boîte postale et un numéro de téléphone ${ }^{80}$. L'Etat providence grec, dans le modèle sud européen ${ }^{81}$, demeure l'un des moins développé en matière de protection sociale pour les nationaux et plus encore pour les exilés ${ }^{82}$. Il n'y avait que deux centres d'accueil de demandeurs d'asile jusqu'en 1997, dont un seul financé par l'Etat (à Lavrio au sud d'Athènes) quand les autres pays européens en comptaient des dizaines. Dans les années 1990, les seules organisations ayant les moyens d'intervenir en faveur des exilés face aux afflux ponctuels étaient des ONG internationales comme Médecins du MondeGrèce, Médecins sans frontières et La Croix Rouge hellénique ${ }^{83}$. A partir de la fin des années 1990, de nouvelles ONG ouvrent des centres d'accueil de petite dimension à 
Athènes, dans l'Attique, près de Thessalonique puis en Crète. Financées sur fonds publics elles sont plus gestionnaires que militantes ${ }^{84}$. L'une des rares organisations militantes et indépendantes est le Réseau de soutien aux immigrés et réfugiés (DIKTIO) qui demeure néanmoins marginal dans l'espace politique. Quelques associations plus récentes apparaissent (Initiative anti-raciste de Thessalonique, association Antigone...), mais elles concernent la lutte contre le racisme et la xénophobie plus que les politiques d'immigration et d'asile. D'autres, plus anciennes, sur les droits de l'Homme, sont peu ou pas spécialisées sur les exilés (Fondation Marangopoulos, Greek Helsinki Monitor \& Minority Rights Group). Les capacités de mobilisation sociale d'un milieu aussi faible ne peuvent être que limitées face aux politiques anti-migratoires qui se dessinent en Grèce depuis quelques années.

Ces deux cas nationaux, s'ils ne sont pas exceptionnels donnent une idée des rapports de forces susceptibles de s'établir au niveau européen. Le problème se pose de manière cruciale notamment depuis l'amorce en 1999, lors du sommet de Tampere, d'un processus d'européanisation des politiques de l'asile et de l'immigration. Avant cette date, la Commission européenne peinait à s'imposer dans ce domaine ce qui laissait au HCR, notamment son Bureau pour l'Europe, un rôle primordial. Ce rôle, le HCR le devait aussi à des relations de forte proximité avec les organisations non gouvernementales dans les différents pays. Ces dernières ont beaucoup espéré d'une européanisation des décisions : la stratégie dite du "boomerang" aurait consisté à impulser à partir du niveau européen des progrès devenus impossibles à obtenir au niveau national. Cependant deux facteurs ont mis cette stratégie en échec. A partir de 2002, le HCR achève de modifier ses positions politiques. En évoluant il prive, en partie au moins, les ONG d'un allié précieux dans un cadre, celui de la politique européenne, où elles sont plus faibles encore qu'au niveau national.

Le HCR est un organisme intergouvernemental formellement rattaché à l'ONU mais politiquement dépendant des gouvernements qui le financent; dans l'ordre des contributions : Etats-Unis, Commission européenne, Japon, Suède, Royaume-Uni, PaysBas, Allemagne, Norvège, Danemark, Canada, Suisse, Finlande, Italie, Australie, France... Les subventions cumulées de contributeurs européens (pays précités + commission), représentent près de la moitié du budget du $\mathrm{HCR}^{85}$. L'Europe forme ainsi une sorte d'actionnaire majoritaire dont les vues, nationales-sécuritaires, s'expriment dans le Comité exécutif qui réunit les Etats financeurs. François Crépeau ${ }^{86}$ souligne l'apparition dès la fin des années 1980 de rhétoriques amalgamant réfugiés, demandeurs d'asile et migrants économiques, restreignant les libertés de déplacement et de choix de destination des exilés, justifiant les mesures répressives des pays finançant le HCR. Dans la décennie suivante, la doctrine du HCR se retourne, note Luc Legoux, passant de la défense du "droit de partir » à celle du «droit de rester ${ }^{87}$. Le HCR développe les rhétoriques de déni d'asile et d'externalisation de l'asile ${ }^{88}$ ainsi que les programmes de " retours volontaires $»^{89}$, souvent forcés ${ }^{90}$. Cette évolution, observe Anne Hammerstad ${ }^{91}$ est bien accueillie par les Etats financeurs qui augmentent massivement le budget du HCR durant la période. Cette tendance déjà très marquée, s'accélère considérablement avec l'arrivée à la tête du HCR, le $1^{\text {er }}$ janvier 2001, de Ruud Lübbers, Premier ministre hollandais de centre droit ayant effectué dans son pays le plus long mandat (12 ans) de chef de gouvernement depuis 1945. Parfaitement en phase avec les préoccupations de ses pairs, chefs de gouvernements européens, Ruud Lübbers achève de subvertir la 
Convention de Genève au sein de son organisation et lui fait jouer un rôle moteur dans la politique européenne des camps ${ }^{92}$.

Les organisations non gouvernementales pouvant intervenir au niveau européen sont rares et faibles : quelques réseaux transnationaux (Migration Policy Group, Migreurop, No Border, Odysseus, Statewatch), des coordinations d'associations (Conseil Européen sur les Réfugiés et l'Exil, Plate-forme pour la Coopération Internationale pour les Migrants Sans-Papiers, Coordination Européenne pour le Droit des Etrangers à vivre en Famille) et des organisations internationales (Amnesty International, Fédération International des Droits de l'Homme) ${ }^{93}$. Mais un regard sociologique vide ce tableau esquissé par simple énumération. Certaines, crées ou financées par les institutions qu'elles sont censées influencer, sont à peine qualifiables de "non gouvernementales » (Odysseus, CERE). D'autres, spécialisées sur un enjeu se limitent à lui dans leurs prises de position (CEDEF) ou, intéressées à un domaine plus large, n'interviennent que ponctuellement sur le secteur (FIDH). D'autres enfin sont marginales (No Border) ou embryonnaires (Migreurop). L'ensemble forme une configuration dominée par des acteurs issus des pays du nord de l'Europe, au style d'action moins protestataire que dans le sud (Italie, France, Espagne...) et plus tournés vers les actions d'influence passant par le dialogue, les pressions et les négociations ${ }^{94}$. La Commission européenne sélectionne de surcroît ces interlocuteurs sur ce critère. Ainsi, sa Communication de $2003^{95}$ relative à l'externalisation de l'asile ne se réfère qu'à deux ONG : Amnesty International, financièrement indépendante tient un discours très critique alors que celui du Conseil Européen sur les Réfugiés et l'Exil, proche de la Commission, l'est beaucoup moins.

\section{La politique des camps : chronique européenne ${ }^{96}$}

61 La politique des camps prolonge les deux mouvements précédemment décrits de stigmatisation du faux-réfugié (spirale du rejet) et de construction de l'exilé comme menace (tournant national-sécuritaire). Dans les premières années du troisième millénaire, émergent des projets politiques visant à bannir et à enfermer dans des camps au bord de l'Europe des exilés déjà entrés ou s'approchant des frontières.

Ces politiques sont moins nouvelles qu'il y paraît : les phénomènes de criminalisation et d'enfermement des migrants sont déjà très avancés à l'intérieur de l'Europe. Ce qu'il y a de nouveau tient au caractère plus explicite des projets de création de camps d'enfermement ou de regroupement forcé, sous des motifs de tri et de dissuasion, d'une part, et à la volonté de les placer dans des pays-tiers qui ne sont pas auteurs des choix politiques ainsi initiés, d'autre part. C'est sur cette nouveauté que sera focalisé le regard en présentant une chronique des délibérations politiques européennes sur le sujet. Un tel choix permet de mettre en évidence certains aspects qui disparaîtraient au contraire dans un panorama plus large mais il implique des renoncements: la "solution pacifique ${ }^{97}$ en Australie, pourtant essentielle tant comme source d'inspiration politique que comme exemple de développements potentiels des projets européens, ne sera pas présentée. En outre, les politiques connexes à celle des camps ne seront pas abordées: les politiques policières de coups de filet à l'encontre des clandestins, les politiques d'expulsions de masse et de transferts internationaux d'exilés accompagnent logiquement celles du regroupement dans des camps délocalisés. Enfin, le développement des clauses anti-migratoires dans les politiques 
nationales de coopération et d'aide au développement des pays pauvres, notamment africains, ne sera pas étudié non plus en soi sauf, in fine, lorsque ces clauses deviennent l'instrument principal de la politique des camps.

Dans le cadre limité de cet article, la focale adoptée permet de faire apparaître l'identité sociologique des acteurs clefs et ainsi l'origine technocratique de ces politiques en distinguant deux processus successifs : l'un de gestation tâtonnante et peu médiatisée, qui impliqua entre septembre 2002 et juillet 2003 des hauts-fonctionnaires et des ministres plutôt discrets. L'autre phase, de janvier à novembre 2004, entraîne une médiatisation forte, tirée par des déclarations ministérielles ostentatoires sur ces projets ainsi que leur institutionnalisation dans les négociations européennes qui aboutirent à une plateforme politique, "Le programme de la Haye ", adopté par le Conseil européen du 5 novembre 2004.

\section{Gestation technocratique : le temps des fonctionnaires (septembre 2002 - juillet} 2003)

L'idée de "traiter" les demandes d'asile au plus loin de l'Europe, dans la région d'origine des exilés, n'est pas nouvelle. Elle apparaît dans les travaux de la Commission européenne dès la fin de l'année $2000^{98}$ mais dans des termes encore vagues. La conceptualisation en a été réalisée par le HCR après l'arrivée, le $1^{\mathrm{er}}$ janvier 2001 , de l'actuel Haut-Commissaire Ruud Lübbers. Le rôle du HCR est essentiel notamment parce qu'il constitua pendant plusieurs décennies la seule instance supranationale à pouvoir harmoniser les politiques de l'asile en Europe. Le HCR joua dans ce secteur le rôle de la Commission européenne longtemps avant que celle-ci ne puisse intervenir: très " européen » tant par son histoire que par ses financements le HCR anime encore aujourd'hui cette gouvernance européenne mais partage progressivement cette fonction d'impulsion avec la Commission notamment depuis le Sommet européen de Tampere (1999).

Le rôle personnel de l'ancien Premier ministre hollandais, Ruud Lübbers, est essentiel aussi sous deux aspects : dans cette organisation très hiérarchisée qu'est le HCR, il détient de facto un quasi-monopole de prise de parole officielle et marque ainsi fortement la communication publique de l'organisation; en outre, son pouvoir de nomination aux postes supérieurs entraîne un alignement progressif d'une partie des cadres supérieurs derrière lui et ses idées. Ce constat ne doit pas occulter cependant l'ampleur des clivages internes notamment celui qui découle des antagonismes entre la volonté des Etats financeurs du HCR d'une part et l'intérêt des réfugiés sollicitant la protection de ces Etats d'autre part. Deux camps, aux périmètres respectifs incertains et mouvants, s'affrontent de manière feutrée au sein du HCR tant au sommet de l'organisation qu'entre les salariés de terrain et les cadres supérieurs. L'année 2001, année de célébration du cinquantenaire de la Convention de Genève sur les réfugiés, gèle provisoirement les contestations de celle-ci et les luttes autour de ses usages. Cette célébration offre l'occasion au HCR de procéder à de vastes consultations internationales et de préparer un texte de politique générale pour les années à venir : "L'Agenda pour la protection ${ }^{99}$. Préparé dans ce contexte d'euphorie commémorative et adopté en juin 2002, ce texte donne satisfaction à toutes les sensibilités internes au HCR : on y trouve ainsi des idées et des orientations tout à fait opposées. Utile pour réunir comme il convient lors d'un anniversaire, malgré des divergences visibles, ce 
texte hétéroclite n'a guère de signification politique globale et, pour cette raison, n'aura pas plus d'incidence politique.

La nouvelle vision politique du HCR s'affirme plus clairement le 13 septembre 2002 dans un discours du Haut-Commissaire Ruud Lübbers devant un Conseil informel «Justices et affaires intérieures » de l'Union européenne à Copenhague. Tout en rappelant son rôle de "gardien de la Convention de 1951 sur les réfugiés ", il la remet explicitement en question, en affirmant «qu'elle ne suffit plus » et en souhaitant une autre approche qu'il nomme "Convention Plus »" ${ }^{100}$. Ce sont les premières idées relatives à ce qu'il nomme alors «la dimension externe des politiques européennes de l'asile». Le dirigeant du HCR propose que dans les cas de "mouvements secondaires» (lorsque des réfugiés quittent leur premier pays de passage pour aller chercheur refuge plus loin) que des accords spéciaux soient établis définissant, au sujet des demandeurs d'asile, les rôles et responsabilités respectifs des pays de destination, de transit mais aussi d'origine, c'està-dire des Etats susceptibles d'être à l'origine des persécutions ayant mis les exilés en fuite. Ruud Lübbers insiste en outre longuement sur l'intérêt des solutions de protection dans les régions d'origine en appelant à augmenter l'assistance nécessaire aux pays pauvres pour répondre aux besoins des réfugiés, assistance qui finance notamment les activités du HCR lui-même en tant que principal organisme gestionnaire de camps dans le monde. Cette présentation de la « Convention Plus » s'achève ainsi:

« En accordant une plus grande attention à la mise en œuvre de solutions durables dans les régions d'origine, le nombre de réfugiés à installer dans les pays européens diminuera et la nécessité d'intégrer ces gens dans vos sociétés sera plus facile à expliquer à vos citoyens. Par dessus tout, le problème des réfugiés tombant dans les mains de contrebandiers et trafiquants d'êtres humains diminuera et les mouvements de réfugiés n'alimenteront plus les réseaux criminels comme il le font aujourd'hui $»^{101}$.

Les initiatives de la Commission européenne et du HCR s'inscrivent dans un contexte de pressions croissantes de la part des gouvernements nationaux et notamment des présidences successives de l'Union européenne. En 2001 plusieurs propositions de directives émanant de la Commission achoppent sous la présidence belge. Représentées un an plus tard, elles marqueront alors un affaiblissement considérable des normes de protection des réfugiés ${ }^{102}$. En 2002 la présidence danoise joue un rôle important dans la promotion des solutions de protection des réfugiés au plus loin de l'Europe, dans les régions d'origine et dialogue à ce sujet avec les gouvernements hollandais et britannique. C'est dans ce contexte qu'apparait début 2003 ce qu'il est convenu d'appeler les "propositions britanniques" quand bien même le gouvernement britannique n'en est pas l'auteur exclusif mais plutôt le premier intéressé à les faire connaître publiquement dans un contexte national d'augmentation rapide du nombre de demandes d'asile et de surenchères xénophobes dans la presse et l'opposition conservatrice. Divulguées en Grande-Bretagne ces propositions ont été élaborées en fait par plusieurs gouvernements nationaux (Royaume-Uni, Pays-Bas, Danemark...), en collaboration avec le HCR et la Commission européenne ${ }^{103}$.

Elles sont révélées au grand public le 5 février 2003 par un article du journal The Guardian ${ }^{104}$ sur la base d'un document «confidentiel» du ministère de l'Intérieur britannique. Le quotidien, à qui a été transmis ce document, fait état d'un plan destiné à faire baisser le nombre de demandeurs d'asile arrivant en Grande-Bretagne en les déportant vers des "aires régionales de protection » situées dans les régions d'origine. Ces zones se situeraient en Turquie, Iran et Kurdistan irakien pour les réfugiés irakiens, 
en Somalie du nord pour les réfugiés du sud de la Somalie et au Maroc pour les Algériens. D'autres zones sont envisagées dans les Balkans et au Zimbabwe ${ }^{105}$. L'Ukraine et la Russie sont également évoquées signalent les journalistes pour mettre un terme aux flux migratoires économiques provenant de l'est de la frontière de l'Europe nouvellement élargie. Présenté officiellement le 10 février 2003 au Haut-Commissaire Ruud Lübbers alors en visite à Londres, le projet prévoit de confier au HCR la tâche et les moyens de gérer ces zones de protection. Aucune réaction publique n'est alors exprimée par le $\mathrm{HCR}^{106}$. Le projet est transmis le 10 mars 2003 par le gouvernement britannique à la présidence grecque de l'Union européenne en vue du sommet européen qui aura lieu le 20 juin 2003 à Thessalonique ${ }^{107}$. Dans sa lettre d'accompagnement, le Premier ministre britannique, Tony Blair, indique que ce projet a été discuté avec le Commissaire européen Vitorino, le Haut-Commissaire Ruud Lübbers et des gouvernements européens. Deux propositions sont exprimées :

1) «Améliorer la gestion régionale. Notre but à long terme devrait être de mieux gérer les migrants irréguliers dans leur région d'origine en : s'attaquant aux causes des flux massifs de population; offrant une meilleure protection aux personnes déplacées près de leur région d'origine et développant des voies légales par lesquelles les vrais réfugiés pourront, si nécessaire, venir en Europe. De telles améliorations pourraient réellement aider ceux qui ont un véritable besoin de protection et permettre aux pays européens de mieux gérer les flux de réfugiés. Cela se ferait en référence aux travaux déjà en cours au HCR (Convention plus) et au sein de l'Union européenne pour poursuivre l'action dans les régions d'origine tel qu'initié par le Conseil européen de Séville (...)»

2) «Centre de transit pour le traitement [des demandes d'asile]. Conjointement à une meilleure protection dans les régions d'origine, il est important, à moyen terme, de dissuader les personnes qui entrent dans l'Union européenne illégalement et qui déposent des demandes non fondées. Une solution envisageable serait d'établir des zones protégées, dans les pays-tiers, vers lesquelles les personnes qui arrivent dans les Etats membres de l'Union et demandent l'asile pourraient être transférées pour que leur demande d'asile soit examinée. Ces centres de 'transit pour le traitement des demandes d'asile' seraient situés sur les voies de transit vers l'Union européenne. Ceux à qui le statut de réfugié serait accordé seraient réinstallés dans les Etats membres participants. Les autres seraient renvoyés dans leur pays d'origine. Cette approche serait à la fois dissuasive pour ceux qui cherchent à abuser du système d'asile tout en préservant le droit à la protection pour ceux qui la méritent vraiment $»^{108}$.

Ce projet fait l'objet de discussions lors du Conseil informel «Justices et affaires intérieures" de l'Union européenne à Véria (Grèce) le 27 et 28 mars 2003. Les gouvernements représentés lors de ce Conseil sont alors loin d'être unanimes face à ces propositions qui donnent lieu à un "vif débat " $^{109}$. Le réseau Migreurop a reconstitué les positions en présence à partir de la presse internationale ${ }^{110}$ : l'Espagne, les Pays-Bas, la Belgique et l'Italie se seraient déclarés en faveur de la proposition britannique tandis que l'Allemagne s'y serait opposée, la France et la Grèce s'abstenant de prendre position, le Portugal, la Finlande et la Suède subordonnant leur positions à l'accord du HCR. Or le Haut-Commissaire Ruud Lübbers, invité à s'exprimer lors de cette rencontre européenne, fait une déclaration très favorable aux propositions britanniques ${ }^{111}$. Il soutient sans réserve la première proposition de zones de protection et, en ce qui concerne la seconde, préconise, lui aussi, la création de «centres fermés " pour les " migrants économiques », majoritaires selon lui, mais suggère que ces camps devraient se situer à l'intérieur des nouvelles frontières européennes plutôt qu'à l'extérieur. 
Cette orientation politique suscite progressivement des réactions d'opposition aussi bien en Grande-Bretagne que dans le reste de l'Europe. Des commentaires très critiques sont faits par l'organisation Statewatch ${ }^{112}$, le Conseil britannique pour les réfugiés ${ }^{113}$, Amnesty International ${ }^{14}$, le Conseil européen pour les Réfugiés et l'Asile (CERE/ $\mathrm{ECRE})^{115}$. La presse internationale elle-même présente ces projets de manière distanciée voire critique. Cependant, l'engagement explicite du Haut-Commissariat aux Réfugiés de l'ONU, traditionnel gardien des intérêts des réfugiés, en faveur de ces solutions leur donnent une légitimité inédite et place les $O N G$ militantes en porte-à-faux vis-à-vis d'un HCR qui constitua longtemps un allié traditionnel au sein des instances gouvernementales. Au sein même du HCR, nombreux sont ceux qui, dans les niveaux inférieurs d'une hiérarchie pesante, font un bilan affligé de l'après Véria : «les experts, les représentants des gouvernements et les médias, sans parler de l'opinion publique, sont complètement désorientés et ne savent plus qui a proposé quoi. Dans ce désastre politique il n'y a pas de vainqueur $»^{116}$.

71 La position du HCR se confirme dès le mois d'avril ${ }^{117}$ avec un document qui commence à circuler deux mois avant d'être rendu public en juin $2003:$ « La proposition d'une triple approche du HCR » (UNHCR's three-pronged proposal) ${ }^{118}$. Il s'agit d'un texte d'application de la «Convention plus» au contexte spécifique de l'Europe. Ce texte plus largement discuté et négocié au sein de l'organisation qu'un simple discours du HautCommissaire, atteste d'une implication plus large de l'encadrement supérieur du HCR dans cette nouvelle orientation politique. La notion d'approche (pronged) a un sens militaire : elle désigne alors les mouvements coordonnés en direction d'une même cible. Ils sont ici au nombre de trois : 1) améliorer les solutions de protection dans la région d'origine en y renvoyant ceux qui en sont partis irrégulièrement (« l'approche régionale ») ; 2) améliorer les systèmes nationaux de l'asile sauf pour tous ceux qui n'y ont pas droit et devront être renvoyés dans leur pays ou le pays de premier accueil ( l'approche nationale ») ; 3) pré-traiter les demandes d'asile dans des centres fermés européens et accentuer les efforts communs d'expulsion des cas manifestement infondés vers les pays d'origine («l'approche européenne»). Présenté initialement comme une contre-proposition aux projets britanniques, la triple approche du HCR en reprend et en valide de facto les principales idées fortes dans un sens "interniste ». Comme le reconnaîtra un porte-parole du HCR, Rupert Colville, le HCR et la Commission européenne travaillent alors à des "propositions parallèles » en faveur de "centres » situés à l'intérieur de l'Union européenne pour traiter les demandes d'asile ${ }^{119}$.

72 A la demande du Conseil, la Commission européenne est amenée à réagir aux propositions britanniques par une Communication qui reprend les propositions du HCR sur le sujet :

«Cette nouvelle approche repose sur trois objectifs spécifiques mais complémentaires, à savoir: 1) l'arrivée organisée dans l'UE, à partir de leur région d'origine, des personnes ayant besoin d'une protection internationale; 2) la répartition des charges et des responsabilités au sein de l'UE ainsi qu'avec les régions d'origine pour qu'elles accordent, dès que possible, une protection effective qui corresponde, autant que faire se peut, aux besoins des personnes ayant besoin d'une protection internationale; 3) la définition d'une approche intégrée en vue de la mise en place, en matière d'asile et de retour, de procédures efficaces débouchant sur des décisions exécutoires $»^{120}$.

73 Trois jours plus tard, une nouvelle réunion du Conseil JAI ${ }^{121}$ (Luxembourg, 05-06 juin 2003) prolonge la discussion sur cette proposition. Le correspondant à Luxembourg du 
journal EU Observer fait état d'un large soutien aux propositions britanniques parmi les gouvernements. Il note l'engagement particulièrement actif des Pays-Bas, du Danemark et de l'Autriche et observe que "l'engagement du Haut-Commissaire aux Réfugiés de l'ONU a indubitablement accru les soutiens à ce projet controversé de l'Union européenne ${ }^{122}$. Dès cette date néanmoins apparaît un obstacle : le veto de la Suède dans un contexte juridique où les décisions doivent encore être prises à l'unanimité. Le ministre suédois des Migrations, Jan 0 . Karlsson fait savoir l'opposition de son gouvernement à une telle politique. Cette position traduit les pressions qui s'exercent, à l'intérieur du pays, sur le gouvernement suédois ${ }^{123}$. Ce veto étant juridiquement insurmontable, soutenu de surcroît par l'Allemagne (soutien affaibli par des désaccords internes ${ }^{124}$ ), les conclusions du Conseil s'annoncent comme un compromis. Le 14 juin déjà, une note interne présente les futures conclusions ${ }^{125}$. Le Conseil demandera à la Commission :
«(...) d'explorer tous les paramètres afin d'assurer une entrée plus ordonnée et mieux gérée des personnes nécessitant une protection internationale, avec la perspective de présenter au Conseil, avant juin 2004, un rapport d'ensemble suggérant des mesures à adopter, et examinant les voies et les moyens d'intensifier la capacité de protection dans les régions d'origine et les pays de premier asile [en particulier sur la base d'une expérience acquise à travers des projets pilotes conduits par les Etats Membres intéressés en partenariat avec les pays concernés et en étroite coopération avec le HCR] et de présenter au Conseil, avant juin 2004, un rapport avec les mesures à prendre leurs implications juridiques $»^{126}$.

La Grande-Bretagne retire alors sa proposition le 16 juin 2003, soit quatre jours avant l'ouverture du sommet européen de Thessalonique ${ }^{127}$. L'extension des soutiens au projet devenant néanmoins de plus en plus évidente, les réactions d'opposition se multiplient durant le mois de juin 2003. Les plus marquantes et probablement les plus influentes ont été celles d'Amnesty International tant à travers son bureau européen à Bruxelles qu'à travers ses délégations nationales. Deux jours avant l'ouverture du Sommet de Thessalonique (et après le revirement britannique), l'ONG publie un document déclarant illégal et impraticable les propositions d'externalisation de l'asile ${ }^{128}$. C'est l'un des moments où Amnesty International attaque le plus directement le HCR placé sur le même plan que le gouvernement britannique et la Commission européenne. La FIDH adresse une lettre ouverte aux gouvernements mais cette missive est plus technique ${ }^{129}$ que celle envoyée le même jour par Amnesty ${ }^{130}$. La conclusion $n^{\circ} 26$ du sommet de Thessalonique sera finalement rédigée ainsi :

«26. Le Conseil européen prend note de la communication de la Commission, qui met l'accent sur des régimes d'asile plus accessibles, plus équitables et mieux gérés, et il invite la Commission à explorer tous les paramètres permettant d'assurer que l'entrée dans l'UE des personnes qui ont besoin d'une protection internationale se fasse d'une manière plus ordonnée et mieux gérée, et à examiner comment les régions d'origine pourraient mieux assurer la protection de ces personnes, en vue de présenter au Conseil, avant juin 2004, un rapport complet proposant des mesures à prendre et leurs conséquences juridiques. Dans le cadre de ce processus, le Conseil européen note qu'un certain nombre d'Etats membres envisagent d'étudier des moyens d'améliorer la protection des réfugiés dans leur région d'origine, en liaison avec le HCR. Ce travail sera effectué en pleine coopération avec les pays concernés sur la base de recommandations du HCR $»^{131}$.

Comparée à la note précitée du 14 juin, cette conclusion fait apparaître dans les deux dernières phrases la volonté de la Commission de ne pas s'impliquer dans la réalisation des fameux "projets-pilotes» (implantations de camps fermés externes) déjà bien avancés. Mais cette conclusion confirme aussi la pérennité de ces projets et leur 
confère une réelle légitimité politique. L'interprétation est assurée par le Premier ministre grec qui, lors de sa conférence de presse, indique que les propositions n'ont pas été repoussées mais " ont été modifiées » et demeurent en suspens ${ }^{132}$. Le même jour le gouvernement britannique, suivant en cela une recommandation de la Commission européenne, fait part de son intention de mettre en œuvre des projets pilotes de camps externes ${ }^{133}$. Le ministre de l'Intérieur britannique annonce ${ }^{134}$ une démarche commune avec des pays partenaires comme les Pays-Bas, le Danemark et l'Autriche pour de telles réalisations en espérant le soutien financier de la Commission et la logistique du HCR. La Commission confirme immédiatement que le projet sera mis en œuvre sous la forme d'expériences pilotes ${ }^{135}$. Le Haut-Commissaire Ruud Lübbers, de son côté, publie un article dans The Guardian pour dénoncer une campagne de manipulation à son encontre et réaffirme l'intérêt de sa "triple approche » ainsi que la nécessité de traiter les demandes d'asile dans des camps fermés à l'intérieur de l'Europe ${ }^{136}$.

Ces « projets-pilotes » avaient déjà donné lieu à des avances diplomatiques de la part du gouvernement britannique en direction notamment de l'Albanie, de la Biélorussie, de la Bulgarie, de la Croatie et de l'Ukraine. L'information est divulguée par The Guardian dès le mois de mars $2003^{137}$, d'abord démentie par le Foreign office puis confirmée par le Home Office au mois de mai ${ }^{138}$ et encore début juin, quelques jours avant le sommet de Thessalonique ${ }^{139}$. Une autre confirmation en est donnée le 7 juillet 2003 lorsque la Croatie et la Bulgarie rejettent publiquement ces projets d'implantation sur leurs territoires respectifs « d'étranges ghettos » où seraient enfermés les exilés ${ }^{140}$.

Un autre résultat de Thessalonique s'esquisse discrètement dès le mois de juillet: la remise en cause du vote à l'unanimité du Conseil, c'est-à-dire la possibilité pour un ou plusieurs Etats, comme l'ont fait la Suède et l'Allemagne, de bloquer des propositions jugées inacceptables. L'enjeu apparaît incidemment moins de deux semaines après le sommet: le 2 juillet la presse fait écho d'une opposition du ministre des Affaires Etrangères allemand à une transformation du mode de décision par adoption du vote à la majorité qualifiée sur ces questions ${ }^{141}$. Ce veto à l'abandon du droit de veto clôt provisoirement le débat, mais provisoirement seulement : le débat sur la procédure de décision sera rouvert un an plus tard et l'opposition du ministre allemand surmontée au cours des négociations du Programme pluriannuel de la Haye.

Après le sommet de Thessalonique, la présidence de l'Union européenne revient à l'Italie qui remet immédiatement sur l'agenda politique ces projets de traitement des demandes d'asile hors du territoire de l'Union européenne ${ }^{142}$. Paradoxalement, cette présidence, par ces excès politiques et ces outrances verbales, freine malgré elle les négociations européennes et fige pendant quelques mois l'avancement des projets d'externalisation auxquelles elle est particulièrement attachée. Le deuxième semestre de l'année 2003 est relativement calme.

\section{Communication politique : le temps des ministres (Janvier 2004-novembre 2004)}

Confirmant son rôle moteur dans cette politique européenne, c'est le HCR qui relance publiquement le débat en janvier 2004 sous la présidence irlandaise. Dans une déclaration ${ }^{143}$ devant le Conseil JAI (à Dublin le 22 janvier 2004), Ruud Lübbers fait état de ses craintes face à un risque d'engorgement des procédures d'asile dans les nouveaux pays rejoignant l'Union européenne du fait de l'application de la Convention de Dublin qui autorise le renvoi des demandeurs d'asile vers le premier pays d'entrée sur le territoire de l'Union. L'argument est simple : si tous les Etats membres de l'Union 
européenne renvoient leurs demandeurs d'asile vers le premier pays d'entrée, les pays situés à la frontière de l'Union seront débordés. Pour éviter cela, il propose non pas une modification de la Convention de Dublin dans un sens plus favorable aux demandeurs d'asile mais, au contraire, les quatre mesures suivantes : 1) la création de "centres de réception européens »; 2) la mise en place d'un mécanisme de "partage du fardeau »; 3) l'établissement d'un système collectif de renvoi rapide des personnes déboutées ; 4) et des avancées en direction d'un système centralisé de traitement des demandes d'asile dans les « centres européens ». En conclusion, indique le communiqué de presse, le Haut Commissaire a déclaré qu'il espérait que la première phase d'harmonisation des législations européennes serait finalisée vers la mi-année 2004. Ces propositions du HCR - qui sont celles de «la triple approche » réactualisée en décembre 2003 pour le volet européen ${ }^{144}$ - devraient pouvoir, a-t-il espéré, constituer une base solide pour la seconde phase d'harmonisation, et contribuer à " une approche européenne vraiment commune pour gérer les flux migratoires irréguliers ».

Faite dans une période d'accalmie, cette relance s'inscrit en effet dans la perspective déjà ouverte de la deuxième étape d'harmonisation des politiques européennes de l'asile et de l'immigration. La première étape correspondant au plan quinquennal adopté lors du Sommet de Tampere en 1999 s'achève officiellement le $1^{\text {er }}$ mai 2004. Dès lors s'ouvrent les négociations du prochain programme pluriannuel d'action dans le domaine de la justice et des affaires intérieures (JAI, rebaptisé «Justice, Libertés et Sécurités» JLS). Ce programme - dit "Programme de la Haye " (voir ci-dessous) adopté le 5 novembre 2004 prévoit l'élaboration d'un "Plan d'action" que la Commission doit proposer au Conseil au printemps 2005. Sur le volet de l'asile et de l'immigration cette deuxième phase s'ouvre avec une communication de la Commission européenne du 4 juin $2004^{145}$ qui fait suite aux conclusions de Thessalonique (juin 2003, conclusion $\mathrm{n}^{\circ} 26$ ). Autorisée par le compromis à ignorer les " projets-pilotes » en cours de réalisation, ce qu'elle rappelle dès la première phrase, la Commission prolonge le commentaire qu'elle avait fait en 2003 du projet britannique et, comme à l'époque, endosse les orientations majeures de ce projet: protection dans les régions d'origine, réinstallation d'exilés hors d'Europe... La Commission reste plus floue dans ses formulations, sur les conditions de mise en œuvre et les dispositifs concrets qui pourraient résulter de ces orientations. Comme l'observe Statewatch: "Là où le gouvernement britannique propose des 'centres externes de traitement' et des 'zones de protection' la Commission utilise des termes plus abstraits se référant aux 'procédures de détermination dans les Etats tiers' et aux 'programmes de protection régionale' $»^{146}$. Cela n'induit cependant aucune modification de sens, aucune inflexion de la politique conduite.

81 Ces deux initiatives technocratiques ne font pas l'objet d'une large publicité. Le sujet ne fait parler de lui, au-delà de la sphère des spécialistes, qu'à partir de l'été 2004. Au début du mois de juillet, trente sept africains embarqués sur un radeau sont sauvés entre la Libye et l'île de Lampedusa (Italie) par le Cap Anamur, bateau appartenant à une ONG allemande. Une controverse politico-médiatique d'ampleur européenne est déclenchée par la réaction brutale du gouvernement italien (long refus d'accostage, mise en accusation des sauveteurs, renvoi des exilés après rejet des demandes d'asile...). Côté allemand, le ministre de l'Intérieur Otto Schilly (social-démocrate), toujours en désaccord avec celui des Affaires Etrangères Joschka Fischer (Verts), saisit l'occasion pour annoncer à ses homologues européens qu'il se rallie à la proposition britannique 
de création de camps d'exilés hors de l'Europe notamment en Afrique du Nord ${ }^{147}$. Il justifie sa décision en disant que "les problèmes de l'Afrique doivent être réglés en Afrique, avec le soutien de l'Europe $»^{148}$. La presse allemande est très critique ${ }^{149}$. Joschka Fischer déclare : «Installer des camps là-bas, je pense que ce n'est pas réfléchi jusqu'au bout » et «je $n^{\prime} e n$ pense rien de bien $»^{150}$. Otto Schilly ne désarme pas et rencontre son homologue italien le 11 août 2004. Ils proposent de créer des " portails de l'immigration » dans les pays d'origine ou de transit. «On ne peut pas laisser les gens se noyer » explique le ministre allemand ${ }^{151}$. Il soutient en outre que "doit être envisagée la création d'une institution européenne, qui, hors des frontières de l'Europe, recevrait et examinerait les demandes d'asile »152. La proposition est aussitôt qualifiée de «bonne idée» par le futur commissaire européen de la Justice, de la Liberté et de la Sécurité, Rocco Buttiglione, ancien ministre des Affaires Européennes de Silvio Berlusconi. Beaucoup de réfugiés pourraient "tout à fait légalement " se rendre en Europe, "si seulement ils savaient comment », affirme-t-il à la Deutschlandfunk, soulignant que ces centres pourraient « informer » les candidats à l'asile ${ }^{153}$.

L'été 2004 est également marqué par une politisation très forte du sujet en Italie et plus largement en Europe en ce qui concerne la politique italienne. Début août un nouveau drame a lieu : soixante-douze exilés partis de Libye sont recueillis après une semaine de dérive par un cargo allemand. Le ministre libyen des Affaires Etrangères fait une déclaration théâtrale à propos de l'invasion de son pays par les immigrés : «Ils sont plus d'un million. S'ils restent encore dix ou quinze ans, la Libye ne sera plus la même. Dans certains quartiers, ils imposent leur loi. (...) On ne sait plus s'ils viennent pour vivre et travailler ou si ce sont des terroristes ». Il ajoute craindre que des extrémistes islamistes ne créent un "Etat-tampon, un royaume islamique avec les rebelles tchadiens dans la région" et ajoute: "seuls, nous n'y arriverons pas " ${ }^{154}$. Les mois d'août, septembre et octobre donnent lieu à une hyper-médiatisation des relations italo-libyenne. Un véritable ballet macabre et diplomatique se déroule rythmé par les arrivées et renvois médiatisés de boat-people, les allers et venues de ministres et de diplomates. La Libye, subitement promue au rang de poste avancée de la lutte européenne contre les exilés, gagne en quelques semaines une réhabilitation politique et diplomatique qu'elle peinait à obtenir depuis des années. L'ancien Etat terroriste devient fréquentable, vertueux même face à la menace migratoire. Le 23 août 2004, Rocco Buttiglione n'hésite pas à parler au sujet de l'immigration de "bombe temporelle " risquant de «submerger " l'Europe ${ }^{155}$; autant d'expressions d'usage courant dans les partis d'extrême droite fait remarquer le directeur de l'observatoire Statewatch ${ }^{156}$. Fin août le Premier ministre italien Silvio Berlusconi se rend en personne à Tripoli et insiste sur la nécessité d'ouvrir des «centres d'accueil » ${ }^{157}$.

83 Parallèlement, le mois de septembre voit fleurir les idées et propositions relatives aux camps extra-européens, preuve s'il en était besoin qu'elles s'acclimatent durablement en Europe. Le ministre suisse de la Justice, Christoph Blocher, propose aussi de créer des camps de réfugiés à l'étranger avec l'aide de l'armée ${ }^{158}$. Le 16 septembre 2004 se tient à Vienne le premier sommet austro-baltique. Autriche, Estonie, Lettonie, Lituanie ont, à cette occasion, l'idée de créer des camps pour tchétchènes en Ukraine ${ }^{159}$. Le gouvernement ukrainien, déjà pressenti par les britanniques, se déclare surpris, fait savoir qu'il trouve l'idée "absurde » et qu'il se demande pourquoi les auteurs de cette idée ont sélectionné l'Ukraine plutôt que leur propre pays ${ }^{160}$. 
84 s'annonce décisif. La presse internationale ${ }^{161}$ identifie les positions en présence: le Royaume-Uni, l'Allemagne, l'Italie, l'Autriche, le Danemark sont pour. La Suède, la France et l'Espagne sont contre, mais pas tous de la même façon: la Suède s'oppose fondamentalement à cette politique alors que la position de la France est ambiguë. Craignant surtout des situations similaires à celle de Sangatte le ministre français de l'Intérieur déclare que "l'immigration d'Afrique subsaharienne est essentiellement économique, et il est hors de question de garder des gens dans des centres". "Il faut les renvoyer dans les pays d'origine et s'attaquer à la racine du problème de l'immigration avec l'ensemble des pays-sources ». Il accepterait néanmoins la création de "points d'accueil » en Afrique du Nord, qui géreraient le retour vers le pays d'origine des «faux" demandeurs d'asile ${ }^{162}$.

Cependant la Commission européenne affiche sa détermination: «M. Vitorino a précisé qu'il n'avait pas besoin de l'accord des gouvernements de l'UE, puisqu'un arrangement existait déjà entre les Nations Unies et la Commission sur ces projets $»^{163}$. Le Haut-Commissaire Ruud Lübbers ne dément pas ${ }^{164}$. Cependant le terme de «camps » commence à disparaître : «Nous avons décidé de ne plus jamais employer ce mot!" affirme Antonio Vitorino ${ }^{165}$. Parallèlement un million d'Euros, co-financés à $80 \%$ par la Commission et à $20 \%$ par les Pays-Bas, sont débloqués pour des «projets-pilotes » en partenariat avec le HCR afin d'aider la Mauritanie, le Maroc, l'Algérie, la Tunisie et la Libye à développer « un système d'asile national ». La notion de camp remarque Alexandrine Bouilhet dans Le Figaro, "a été remplacée par celle de 'centre d'accueil', de 'centre de protection' ou 'centre d'assistance', sans que personne ne sache très bien expliquer la différence ${ }^{166}$. De fait, la France et l'Espagne, tout en affichant une opposition largement relayée par la presse française aux propositions germano-italiennes, prônent lors d'une nouvelle réunion le 17 octobre 2004 la mise en place de "points de contact» dans les pays de transit, principalement d'Afrique du Nord, d'où pourraient être examinées et traitées les demandes d'asile ${ }^{167}$. Cette position ne fait pas obstacle à la poursuite des négociations.

Durant cette même période - de juin à novembre 2004 - se déroule une négociation qui porte sur le programme pluriannuel d'action de l'Union européenne dans le domaine «Justice, Libertés et Sécurités» (JLS). Ce programme, intitulé «Le programme de la Haye : renforcer la liberté, la sécurité et la justice dans l'Union européenne » a été adopté lors du Conseil européen du 5 novembre 2004 à la Haye. Deux semaines avant, un «brouillon» officiel (validé par le COREPER du 14.10.04) est rendu public par l'observatoire Statewatch qui le diffuse le 20 octobre $^{168}$ et souligne que les délais d'adoption ont pour effet de marginaliser les acteurs parlementaires et non gouvernementaux dans le débat politique relatif à ce programme ${ }^{169}$. Ce programme adopté pour cinq ans (01.05.2004 - 01.05.2009) constitue une plateforme politique d'orientation et de coordination des actions européennes dans les multiples domaines du Conseil JLS. Le "Programme de la Haye ", tant par son statut que par ses contenus, marque l'apogée et l'aboutissement de cette phase «publique» de la politique. Il est d'ailleurs possible que la médiatisation des enjeux migratoires, durant l'été 2004, ait été tirée par les stratégies de négociation de ce programme : les acteurs nationaux qui ont poussé à cette médiatisation, notamment le gouvernement italien et les ministres de l'Intérieur italiens et allemands, ayant pu cherché à peser ainsi sur l'orientation générale du programme. 
87 Le «Programme de la Haye » reflète et illustre le tournant national-sécuritaire: l'introduction procède aux amalgames déjà signalés en appelant à une "approche commune plus efficace des problèmes transfrontaliers tels que l'immigration illégale, la traite des êtres humains, le terrorisme et la criminalité organisée ${ }^{170}$. Parmi les objectifs multiples et contradictoires référés à la liberté, à la sécurité et à la justice, l'introduction met en avant comme priorité la prévention du terrorisme ${ }^{171}$. Au-delà de cette introduction et d'une partie d'orientation générale, le programme aborde trois grands chapitres respectivement consacrés à la liberté, la sécurité et à la justice. Or le chapitre «Renforcer la Liberté » est presque intégralement consacré à la régulation des flux migratoires: après une courte section sur la liberté de circulation des citoyens européens, les six autres sections portent respectivement sur «Politique dans le domaine de l'asile, de l'immigration et des frontières ", "Un régime d'asile européen commun », « Immigration légale et lutte contre l'emploi clandestin », « Intégration des ressortissants de pays-tiers », "La dimension extérieure de l'asile et de l'immigration » et "Gestion des flux migratoires ». A ce cadrage très particulier de la politique de «Liberté », s'ajoute la focalisation sur les enjeux transfrontières des chapitres «Sécurité » et « Justice » qui, de ce fait, recoupent fréquemment le thème migratoire. A l'examen des composantes et proportions du texte, l'enjeu migratoire paraît aussi prioritaire que celui du terrorisme et les deux semblent devoir souvent s'entremêler.

Le Programme de la Haye reflète l'état des discussions engagées depuis septembre 2002 notamment par les propositions du Haut-Commissaire Ruud Lübbers. Le thème de l'asile au plus près du point de départ apparaît sous la forme d'une orientation générale visant à encourager « l'accès à la protection et à des solutions durables au stade le plus précoce. » (\$ 1.6.1) et d'une invitation adressée à la Commission à...

«...élaborer des programmes de protection régionaux de l'UE en partenariat avec les pays-tiers concernés et en étroite consultation et coopération avec le Haut Commissariat des Nations Unies pour les réfugiés. Ces programmes s'inspireront de l'expérience acquise lors de la mise en œuvre des programmes de protection pilotes qui seront lancés avant la fin 2005. Ils utiliseront différents instruments pertinents, centrés essentiellement sur le renforcement des capacités, et comprendront un programme commun de réinstallation pour les Etats membres qui souhaitent y participer ». (§ 1.6.2)

89 Cette invitation met en œuvre la proposition des «zones de protection spéciale » imaginée en 2003 par le Premier ministre britannique et validée par le HCR ; elle offre surtout un cadre politique aux "projets pilotes" officialisés un mois plus tôt et contraint ainsi la Commission, réticente, à accompagner le développement de ces "projets pilotes» en voie de généralisation et d'institutionnalisation. En ce qui concerne les «instruments pertinents » il n'est plus jamais fait mention du mot "camp», comme l'a annoncé Antonio Vittorino un mois plus tôt. L'ensemble du Programme de la Haye est rédigé en substituant le mot "capacité »: on parle de «capacité de gestion migratoire ", de "capacité de contrôle des frontières » et de « capacité de protection des réfugiés ». La finalité générale est explicite :

«L'asile et l'immigration sont, par nature, des questions internationales. La politique de l'UE devrait viser à soutenir, dans le cadre d'un véritable partenariat et en recourant, le cas échéant, aux fonds communautaires existants, les efforts déployés par les pays-tiers pour améliorer leur capacité à gérer les migrations et à protéger les réfugiés, pour prévenir et combattre l'immigration clandestine, pour informer sur les voies légales de migration, pour régler la situation des réfugiés en leur offrant un meilleurs accès à des solutions durables, pour renforcer les moyens 
de surveillance des frontières, pour améliorer la sécurité des documents et pour

s'attaquer au problème du retour ». (§ 1.6.1)

Si on cherche à identifier en quoi consistent ces «capacités » on s'aperçoit qu'elles répondent à deux fonctions principales: empêcher les exilés d'arriver en Europe (§ 1.6.3.) et recevoir ceux qui sont renvoyés d'Europe (\$ 1.6.4.). Le mot "capacité", comme substitue du mot " camps », n'est pas un simple euphémisme technocratique : il exprime une évolution dans l'approche européenne du sujet. Après deux ans de tâtonnements, la trajectoire de "bonne " gouvernance semble trouvée qui consiste, sur le modèle des directives aux Etats membres, à fixer des objectifs anti-migratoires aux Etats tiers, dits " partenaires ", en leur laissant la liberté et la responsabilité éthique et politique du choix des moyens. D'une certaine manière l'Union européenne ne leur impose « que » des obligations de résultats dans la lutte anti-migratoire sans regarder au choix des moyens et tout en acceptant de contribuer au financement de ceux-ci.

Deux séries d'instruments de politique publique européenne sont envisagées pour parvenir à ce renforcement des «capacités» mises en œuvre par les Etats «partenaires » dans la lutte anti-migratoire :

92 1) l'un, généraliste, consiste à placer les enjeux migratoires au cœur des relations avec les pays-tiers ( $\$ 1.6 .1$ ) ce qui inclut les relations de coopération économique, d'aide au développement et d'action humanitaire (\$ 1.6.2.) et passe également par une politique dite de « voisinage et de partenariat » en cours d'élaboration (§ 1.6.3) :

«Le Conseil européen engage le Conseil et la Commission à poursuivre le processus d'intégration complète de la question de l'immigration dans les relations existantes et futures de l'UE avec les pays-tiers. Il invite la Commission à achever, d'ici le printemps 2005, l'intégration de la question de l'immigration dans les documents de stratégie par pays et par région pour tous les pays-tiers concernés ». (§ 1.6.1)

«Il convient que les politiques qui concernent à la fois l'immigration, la coopération au développement et l'aide humanitaire soient cohérentes et mises au point dans le cadre d'un partenariat et d'un dialogue avec les pays et régions d'origine ». (\$ 1.6.2)

«La proposition de règlement concernant la création d'un instrument européen de voisinage et de partenariat fournit le cadre stratégique pour l'intensification de la coopération et du dialogue en matière d'asile et d'immigration avec les pays voisins, notamment ceux du bassin méditerranéen, ainsi que pour l'élaboration de nouvelles mesures. A cet égard, le Conseil européen demande qu'un rapport lui soit transmis avant a fin de 2005 sur les progrès réalisés et les résultats obtenus ». ( $\S$ 1.6.3)

93 2) l'autre, plus sectoriel, consiste à financer les pays les plus motivés pour accroître leurs « capacités » de régulation des flux migratoires :

«En ce qui concerne les pays de transit, le Conseil européen souligne qu'il faut intensifier la coopération et le renforcement des capacités aux frontières méridionales et orientales de l'UE, afin de permettre aux dits pays de mieux gérer les migrations et d'offrir une protection adéquate aux réfugiés. Les pays qui font preuve d'une réelle volonté de s'acquitter des obligations qui leur incombent en vertu de la Convention de Genève relative au statut des réfugiés se verront offrir une aide visant à renforcer les capacités de leur régime national d'asile et les contrôles aux frontières ainsi qu'une plus vaste coopération en matière de migration ». (§ 1.6.3.)

Ce Programme de la Haye servira de cadre politique général pour les cinq prochaines années (2005-2010) et l'année 2005 sera décisive dans la concrétisation des orientations générales qui tendent à amener les pays voisins de l'Europe à développer par eux- 
mêmes les camps d'enfermement et de regroupement forcé des exilés. A l'abri des mobilisations sociales, des focalisations médiatiques et des controverses publiques intra-européennes les camps devraient se développer sous la responsabilité et sous les régimes politiques et juridiques des Etats voisins. Cette sous-traitance imposée de la lutte anti-migratoire passera pour l'Union européenne par ses politiques étrangères et notamment celles de coopération économique, d'aide au développement et d'action humanitaire subordonnées à cet impératif anti-migratoire.

\section{NOTES}

1. Sur l'usage du mot «camp » dans cet article, on se reportera à l'introduction de ce numéro de la revue Cultures \& Conflits et, dans ce même numéro, à l'article de Caroline Intrand et PierreArnaud Perrouty ainsi qu'à la carte de Migreurop.

2. Le terme « exilé » désignera dans cet article toute personne forcée de migrer et de vivre hors de sa patrie : « demandeurs d'asile », "réfugiés », " déboutés du droit d'asile », " sans-papiers »... Cette notion permet de les désigner ensemble sans dépendre des catégories juridiques ou usuelles dont la genèse et les utilisations constituent un objet de recherche.

3. Noiriel G., Réfugiés et sans-papiers - La République face au droit d'asile, XIX ème $-X^{\text {ème }}$ siècle, Paris, Hachette / Pluriel, 1999, p. 229 et suivantes.

4. Lindblom C.E., The policy-making process (1968), Englewood Cliffs (New-Jersey), Prentice-Hall Inc., 1980 ;Parsons W., Public Policy - An Introduction to the Theory and Practice of Policy Analysis, Aldershot (UK), Brookfield (US), Edward Elgar, 1995.

5. Berger P., Luckmann T.., La construction sociale de la réalité, Paris, Méridiens-Klincksieck, 1986 ; kingston P.W., Nock S.L, The Sociology of Public Issues, Belmont (US), Wadsworth Publishing Company, 1990 ;Rochefort D., Cobb R.W., The Politics of Problem Definition, Lawrence, University Press of Kansas, 1994 ; Giusberg L., Understandig Social Problems, Policies and Programs, (1994), Columbia, University of South California Press, 1999.

6. Agier M., Au bords du monde, les réfugiés, Paris, Flammarion, 2002 ; Castles S., Crawley H., Loughna S., States of Conflict: Causes and patterns of forced migration to the EU and policy responses, London, Institute for Public Policy Research, 2003 ; Haut Commissariat des Nations Unies pour les Réfugiés (HCR), Les réfugiés dans le monde - Cinquante ans d'action humanitaire, Paris, Editions Autrement / HCR, 2000.

7. Voir aussi : Guichaoua A. (dir.), Exilés, réfugiés, déplacées en Afrique centrale et orientale, Paris, Karthala, 2004.

8. Cambrézy L., Réfugiés et exilés - Crise des sociétés, crise des territoires, Paris, Ed. des Archives Contemporaines, 2001.

9. $\mathrm{HCR}$, Les réfugiés dans le monde, op. cit., p. 125.

10. Suède $1,75 \%$ - Danemark $1,3 \%$ - Allemagne $1,19 \%$ - Suisse $1,11 \%$ - Norvège $1,05 \%$ Autriche $1 \%$ - Pays-Bas 0,8 \% - Canada 0,4 \%.

11. Le nombre de demandeurs d'asile depuis 1980 se situe entre 20000 et 180000 par an pour l'ensemble de l'Amérique du Nord et demeure inférieur à 50000 par an pour l'Australie et la Nouvelle-Zélande réunies. 
12. Les chiffres du HCR se rapportent aux pays suivants : Autriche, Belgique, Danemark, Finlande, France, Allemagne, Grèce, Italie, Pays-Bas, Norvège, Espagne, Suède, Suisse, Royaume-Uni. HCR, op. cit., p. 157

13. Durant cette période, le nombre de personnes entrant sous la protection du statut de réfugié en France est de 238000 et le nombre de personne en sortant (décès ou départ ou retrait du statut...) est de 192000 , soit $80,67 \%$ du chiffre précédent, engendrant une croissance de la population totale de seulement 46000 personnes. Voir Legoux L., La crise de l'asile politique en France, Paris, CEPED, 1995, pp. 128-129.

14. Voir Piersons P., "When Effect becomes Cause. Policy Feedback and Political Change", World Politics, 1993, n 45, pp.595-628; Dismantling the Welfare States. Reagan, Thatcher and the Politics of Retrenchment, Cambridge University Press, 1994.

15. Les modalités d'accès aux sources et autres indications méthodologiques seront données au début de chacune des parties.

16. Voir les travaux de Jean-Pierre Masse, L'exception indochinoise. Le dispositif d'accueil des réfugiés politiques en France, 1973-1991, Thèse de doctorat en sociologie politique, Dir. G. Noiriel, Paris, EHESS, 1996, 424 p. ; «L'institutionnalisation de l'accueil, Vers une gestion collective des réfugiés dans Les réfugiés en France et en Europe ", Quarante ans d'application de la convention de Genève, actes du colloque organisé à l'initiative de l'OFPRA les 11-13 juin 1992, décembre 1993, pp. 366-379. "Genèse et institutionnalisation du dispositif d'accueil des réfugiés politiques en France", Cahiers de la sécurité intérieure, $\mathrm{n}^{\circ} 45$, novembre 2001, pp. 45-68.

17. L'auteur a été juge à la Commission des Recours des Réfugiés (CRR) en France, en tant qu'assesseur nommé par le HCR, de janvier 2001 à septembre 2004. Cette activité à temps partiel, menée parallèlement à son activité d'universitaire, l'a amené à participer aux décisions relatives à environ 2500 demandes d'asile. Il s'exprime ici en tant qu'universitaire et ses propos n'engagent ni le HCR ni la CRR. Sur les aspects juridiques de la procédure en France on se reportera à l'article de Valluy J., «La fiction juridique de l'asile ", Pleins Droits, $\mathrm{n}^{\circ}$ 63, décembre 2004.

18. Voir ci-dessous : Le tournant national-sécuritaire : l'exilé comme menace.

19. Il n'est pas possible dans le cadre de cet article d'aborder tous les aspects du processus de rejet et tous les facteurs qui viennent rendre cette spirale du rejet plus irréversible. On se bornera donc à mettre en avant un facteur, celui de la temporalité, qui est important mais qui n'est pas le seul.

20. Sur la procédure en France voir : Decourcelle A., Julinet S., Que reste-t-il du droit d'asile?, Paris, L'esprit frappeur, 2000 ; Bleriot I., Devenir réfugié: entre légalité et pitié, Mémoire de DEA, EHESS (Ecole Doctorale d'Anthropologie sociale et d'Ethnologie), Dir. M. Abélès, Rap. de soutenance : D. Fassin, juin 2003, 117 p.

21. Près d'un tiers des demandes d'asile au Royaume-Uni sont très officiellement rejetées à ce stade de la procédure: Home Office, Asylum Statistics: First Quater 2002 United Kingdom, Londres, Immigration Research and Statistics Services, 2002, p. 14.

22. En Espagne, plus de $70 \%$ des demandes sont rejetées à ce stade de la procédure. A ce sujet, voir Jubany-Baucells O., "The state of welfare for asylum seekers and refugees in Spain ", Critical Social Policy, vol. 22, $\mathrm{n}^{\circ} 3$, p. 420.

23. Blommaert J., "Investigating narrative inequality: African asylum seekers' stories in Belgium ", Discourse \& Society, vol. 12, $\mathrm{n}^{\circ} 4$, pp. 413-449.

24. Amnesty International, Observation des audiences publiques de la Commission des Recours des Réfugiés - Compte-rendu et recommandations, octobre 2003.

25. Doornbos N., "Separated Worlds. The Assessment of Credibility in Claims for Asylum ", Amsterdams Sociologisch Tijdschrift, vol. 31, n 1, 2004, pp. 80-112.

26. Cwerner S.B., "Faster, Faster and Faster - The time politics of asylum in UK", Time \& Society, vol. 13, n 1, 2004, pp. 71-88. 
27. Pour une tentative voir Coffey G., "The Credibility of Evidence at the Refugee Review Tribunal ", International Journal of Refugee Law, vol. 15, $\mathrm{n}^{\circ} 3$, juillet 2003, pp. 377-417.

28. Le marquage politique de "réfugié " pouvait être gênant tant en exil que dans la patrie. Johanna siméant rappelle le nombre de ces militants qui «au lieu de passer par la filière de la demande d'asile, de la moitié des années soixante-dix à la moitié des années quatre-vingt, ont continué à profiter des possibilités d'une législation sur l'immigration - et non l'asile - par encore trop rigide malgré l'arrêt officiel de l'immigration de travail en 1974, alors qu'ils auraient pu en toute légitimité, demander et obtenir l'asile politique ", in Siméant J., La cause des sans-papiers, Paris, Presses de Science Po, 1998, p. 90.

29. Hardy C., "Refugee determination - Power and Resistance in Systems of Foucaldian Power", Administration and Society, vol. $35, \mathrm{n}^{\circ} 4$, septembre 2003, pp. 462-488.

30. Holzer T., Schneider G., Widmer T., "Discriminating Decentralization - Federalism and the handling of asylum applications in Switzerland, 1988-1996 ", Journal of Conflict Resolution, vol. 44, n² 2, avril 2000, pp. 250-276.

31. Sur les statistiques françaises, voir Legoux L., La crise de l'asile politique en France, Paris, Centre Français sur la population et le développement (CEPED), 1995.

32. Tableau élaboré en inversant les proportions présentées, à partir des données du HCR, par Holzer T., Schneider G., Widmer T., «Discriminating Decentralization - Federalism and the handling of asylum applications in Switzerland, 1988-1996», Journal of Conflict Resolution, vol. 44, n² 2, avril 2000, p. 253.

33. Deux chiffres sont indiqués pour les pays qui connaissent deux niveaux de procédure (première instance et appel). Ils sont produits à partir des données de l'UNHCR, « 2003 Global Refugee Trends - Overview of Refugee Populations, New arrivals, durable solutions, asylum-seekers and other persons of concern to UNHCR », 15 juin 2004. Les pays marqués d'une étoile connaissent une deuxième sorte de protection des réfugiés, alternative à la Convention de Genève: les "protections subsidiaires", précaires et de courtes durées, ne permettent pas au réfugié d'envisager de refaire sa vie dans le pays d'accueil.

34. Document du gouvernement britannique intitulé «New International Approaches to Asylum Processing and Protection » accompagnant la lettre de Tony Blair à Costas Simitis du 10 mars 2003.

35. Wintour P. (Chief political correspondent), «Blair warning on rights treaty», The Guardian, 27 janvier 2003.

36. Assemblée Nationale, Débats Parlementaires, Journal Officiel de la République Française du vendredi 6 juin 2003; Compte-rendu intégral de la séance du jeudi 5 juin 2003, Intervention de M. Dominique de Villepin, ministre des Affaires Etrangères.

37. Communication de la Commission au Conseil et au Parlement européen, «Vers une procédure d'asile commune et un statut uniforme, valable dans toute l'Union, pour les personnes qui se voient accorder l'asile », COM/2000/0755 final.

38. House of Commons, Home Affairs Committee, Asylum removals, Fourth Report of Session 2002-03, vol. I : Report and Proceedings of the Committee, Londres, The Stationery Office Limited, 8 mai 2003, p. 7.

39. Cité par Travis A., « UN puts forwards 'fairer' alternative to Blunkett's asylum processing plan », The Guardian, 12 mai 2003.

40. "J'ai grandement élargi les possibilités de venir via les voies légales pour les immigrants qui travaillent dur. Mais le système du droit d'asile ne peut fonctionner au bénéfice des réfugiés authentiques si on en abuse par ailleurs ", in "Londres se prépare à durcir son droit d'asile », Dépêche AFP, lundi 27 octobre 2003, $13 \mathrm{~h} 39$.

41. Communication de la Commission au Conseil et au Parlement sur « la politique commune d'asile et l'Agenda pour la protection ", (Deuxième rapport de la Commission sur la mise en oeuvre de la COM(2000)755 final du 22 novembre 2000), Bruxelles, le 26 mars 2003. COM(2003)152 final, p. 3. 
42. Guild E., "International terrorism and EU immigration: Asylum and borders policy: the unexpected victims of 11 September 2001 », European Foreign Affairs September 2001, automne 2003, vol. 8, n 3, pp. 331-346.

43. Crépeau F., Droit d'asile - De l'hospitalité aux contrôles migratoires, Bruxelles, Ed. Bruylant / Ed. de l'Université de Bruxelles, 1995, p. 254.

44. Voir les conclusions du Comité Exécutif du HCR de l'ONU : EXCOM nº 30 (XXXIV/1983) relative au problème des demandes manifestement infondées ou abusives du statut de réfugié ou d'asile.

45. Voir Crépeau F., Droit d'asile..., op. cit, p. 266 et suivantes.

46. La sociologue du politique Annie Collovald vient de déconstruire efficacement le mécanisme très élitiste de qualification de l'extrême droite par référence au peuple et a montré, dans le cas de la France, que la base sociale tant des électeurs que des leaders de cette sensibilité n'a rien de spécifiquement populaire. Collovald A., Le "Populisme du FN», un dangereux contresens, Paris, Editions du Croquant, 2004.

47. Van Leeuwen T., Wodak R., «Legitimizing immigration control: discourse-historical analysis ", Discourse Studies, 1999, vol. 1, n 1, pp. 83-118.

48. Calavita K., "A 'reserve army of delinquents' - The criminalization and economic punishment of immigrants in Spain », Punishment \& Society, 2003, vol. 5, n 4, pp. 399-413.

49. Lochak D., "La politique de l'immigration au prisme de la législation sur les étrangers ", in Fassin D., Morice A., Quiminal C.(dir.), Les lois de l'inhospitalité: les politiques de l'immigration à l'épreuve des sans-papiers, Paris, La Découverte, 1997.

50. Tsoukala A., "Le contrôle de l'immigration en Grèce dans les années quatre-vingt-dix », Cultures \& Conflits, 1997, $\mathrm{n}^{\circ}$ 26/27, pp. 51-72.

51. Angel-Ajani A., "Italy's racial cauldron - Immigration, Criminalization and the Cultural Politics of Race ", Cultural Dynamics, 2000, vol.12, n³, pp.331-352 ; "A question of dangerous race?", Punishment \& Society, 2003, vol. 5, $\mathrm{n}^{\circ}$ 4, pp. 433-448; Melossi D., «In peaceful life - Migration and the crime of modernity in Europe/Italy », Punishment \& Society, 2003, vol. 5, n 4, pp. 371-397.

52. Cohen S., "The local state of immigration controls", Critical Social Policy, 2002, vol. 22, $\mathrm{n}^{\circ} 3$, pp. 518-543 ; Fekete L., " The emergence of xeno-racism », Race \& Class, 2001, vol. 43, n², pp. 23-40 ; Schuster L., Solomos J., "Race, immigration and asylum - New Labour's and its consequences", Ethnicities, 2004, vol. 46, nº 2, pp. 267-300.

53. Cette partie est fondée sur la bibliographie citée en référence et sur deux enquêtes spécifiques : 1) En France les observations ont été réalisées entre janvier 2003 et octobre 2004, au moyen de plusieurs dizaines d'entretiens informels auprès de responsables associatifs à Paris et à Lyon, d'un suivi des assises de la Confédération Nationale du Droit d'Asile (CFDA) et d'un suivi de la presse spécialisée et des supports Internet très utilisés par les réseaux associatifs. 2) En Grèce les observations proviennent d'une série de quinze entretiens semi-directifs réalisés à Athènes en décembre 2003 auprès de responsables et militants associatifs ainsi que d'universitaires spécialisés sur ces questions.

54. Lochak D., « La politique de l'immigration au prisme de la législation sur les étrangers, op. cit.

55. Vasta E., "Rights and Racism in a New Country of Immigration: The Italian Case ", in Solomos J., Wrench J. (dir.), Racism and Migration in Western Europe, Oxford, Berg Publishers, 1993, pp. 83-98.

56. Angel-Ajani A., «Italy's racial cauldron - Immigration, Criminalization and the Cultural Politics of Race », op. cit., p. 338.

57. Wodak R., "The Genesis of Racist Discourse in Austria since 1989 ", in Caldas-Coulthard C.R., Coulthard M., (dir.), Texts and Practices - Readings in Critical Discourse Analysis, London, Routledge, 1996, pp. 62-95.

58. Matouschek B., Wodak R., Januschek F., Notwendig Maßnahmen gegen Fremde?, Vienne, Passagenverlag, 1995. 
59. Van Leeuwen T., Wodak R., "Legitimizing immigration control: a discourse-historical analysis ", Discourse Studies, 1999, vol. 1, n 1, pp. 83-118.

60. Renton D., "Examining the success of the British National Party, 1999-2003 ", Race \& Class, 2004, vol. $45, n^{\circ} 2$, pp. $75-85$.

61. Sur l'ensemble du pays, la percée de l'extrême droite reste très modeste : 3 sièges sur 6000 sièges locaux en 2003.

62. Dans la même période il se situe entre 90000 et 440000 en Allemagne.

63. Kaye R., "Defining the Agenda: British Refugee Policy and the Rote of Parties ", Journal of Refugee Studies, 1994, vol. 7, n²/3, pp. 144-159.

64. Kaye R., "Redefining the Refugee: The Media Portrayal of Asylum Seekers », in Koser K., Lutz H. (dir.), The New Migration in Europe: Social Constructions and Social Realities, New-York, St Martin's Press, pp. 163-183.

65. Cohen R., Frontiers of Identity: The British and the Others, London, Longman, 1994.

66. Renton D., « Examining the success of the British National Party, 1999-2003», op. cit., p. 77.

67. Anderson M., "Les frontières : un débat contemporain ", Cultures \& Conflits, 1997, n²6/27, p. 23.

68. Bigo D., "Sécurité et immigration : vers une gouvernementalité par l'inquiétude?", Cultures \& Conflits, 1998, n³1-32, pp. 13-38. Voir aussi à ce sujet et du même auteur : Polices en réseaux. L'expérience européenne, Presses de Sciences Po, Paris, 1996, notamment le chapitre V.

69. . Tsoukala A., « La criminalisation des immigrés en Europe », in Sainati G., Bonelli L., (dir.), La machine à punir, Paris, L'esprit Frappeur, 2000, p. 251.

70. Le phénomène est particulièrement net pour les quatre pays de l'Europe du sud. Voir Calavitta K., «Immigration, law and marginalization in a global economy : notes from Spain », Law and Society Review, 1998, vol. 32, n 3, pp. 529-566.

71. Albrecht H.-J., «Ethnic minorities and crime - the construction of foreigners'crime in the Federal Republic of Germany », in Palidda S., Délit d'immigration, COST A2 Migrations, Commission européenne, Bruxelles 1997, p. 99. Cité dans Tsoukala A., «La criminalisation des immigrés en Europe ", op. cit., p. 254.

72. Lynch J.P., Simon R.J., «A comparative assessment of criminal involvement among immigrants and natives across seven nations ", International Criminal Justice Review, 1999, n 9 , pp. 1-17.

73. Données issues du Conseil de l'Europe, présentées par Martin Baldwin-Edwards, "Semireluctant hosts : Southern Europe's Ambivalent Response to Immigration », Panteion University, Research Institute of Environmental and Human Resources, Mediterranean Migration Observatory, MMO Working Paper No. 3, novembre 2001, p. 7.

74. Melossy D., «In a peaceful life - Migration and the crime of modernity in Europe/Italy », op. cit., p. 377.

75. Baldwin-Edwards M., «Semi-reluctant hosts: Southern Europe's Ambivalent Response to Immigration », op. cit., p. 7.

76. Den Boer M., « Crime et immigration dans l'Union européenne », op. cit., p. 62.

77. Ces remarques ont fait l'objet d'une communication antérieure : Valluy J., «La construction sociale du 'faux-réfugié', entre droit d'asile et politique migratoire », Communication au colloque «Les Droits de l'Homme à l'épreuve de l'immigration et de l'asile », Université Lumières (Lyon 2) / Institut des Sciences de l'Homme, Lyon, le 2 avril 2004.

78. . Il s'agit particulièrement du Service social d'aide aux exilés (SSAE), de l'association France Terre d'Asile (FTDA) et de l'association Forum Réfugiés (FR).

79. Baldwin-Edwards M., «Semi-reluctant hosts: Southern Europe's Ambivalent Response to Immigration », op. cit., p. 3

80. Notre entretien avec Ahmet Mdawia, Président du Forum Grec des Migrants, Athènes, le 24 décembre 2003. 
81. Ferrera M., "The Southern model of Welfare in social Europe », Journal of European Social Policy, 1996, 6 (1) ; "Modèles de solidarité, divergences, convergences: perspectives pour l'Europe ", Revue Suisse de Science Politique, 1996, vol. 2, n 1, pp. 55-72.

82. Sitaropoulos N., «Refugee Welfare in Greece : towards a remodelling of the responsabilityshifting paradigm? ?, Critical Social Policy, 2002, vol. 22, n 3, pp. 436-455.

83. Sitaropoulos N., " Modern Greek Asylum Policy and Practice in the Context of the Relevant European Developments », Journal of Refugee Studies, 2000, vol. 13, n 1, pp. 139-152.

84. Papadopoulou A., "Asylum, Transit Migration and the Politics of Reception: the Case of Kurds in Greece ", Paper prepared for the 1st PhD LSE Symposium on Modern Greece "Current Social Science Research in Greece', 21 juin 2003.

85. Haut Commissariat des Nations Unies pour les Réfugiés (HCR), Les réfugiés dans le monde Cinquante ans d'action humanitaire, op. cit., p. 166.

86. Crépeau F., op. cit., p. 118.

87. Legoux L., "Du 'droit de partir' au 'droit de rester'", Communication à la première Conférence publique du réseau T.E.R.R.A., Paris, Université Panthéon-Sorbonne (Paris 1) le 7 mai 2003.

88. Voir ci-dessus, Le déclin des soutiens aux exilés.

89. Chimni B.S., "The meaning of words and the role of UNHCR in voluntary repatriation", International Journal of Refugee Law, 1993 vol. 5 (3), pp. 442-460. Voir aussi Kendle A.B., « Protecting whom ? : the UNHCR in Sri Lanka, 1987-1997 »,Round Table, n³48, octobre 1998, pp. 521-541.

90. Preston R., "Researching Repatriation and Reconstruction: Who is Researching What and Why?", in Black R., Koser K. (dir.), The End of the Refugee Cycle? - Refugee, Repatriation and Reconstruction, Oxford, Berghahn, 1999, p. 19.

91. Hammerstad A., «Whose Security? UNHCR, Refugee Protection and State Security After the Cold War ", Security Dialogue, 2000, vol. 31, n 1, pp. 391-403.

92. Voir ci-dessous le chapitre intitulé « La politique de camps : chronique européenne ».

93. Monforte P., «Approche comparée des réseaux transnationaux mobilisés autour des politiques d'immigration et d'asile au sein de l'Union européenne », Communication à la conférence publique "L'adaptation des ONG à l'européanisation des politiques de l'asile et de l'immigration », T.E.R.R.A., Université Paris 1, 24 mai 2004.

94. Bouteillet-Paquet D., (Amnesty International - Bureau de Bruxelles), «Evolution du travail d'AI face à l'émergence de politiques européennes d'asile et d'immigration: les techniques de lobbying mises en oeuvre dans un cadre régional ", Communication à la conférence "L'adaptation des ONG à l'européanisation des politiques de l'asile et de l'immigration", T.E.R.R.A., Université Paris 1, 24 mai 2004.

95. Commission européenne, «Communication de la Commission au Conseil et au Parlement européen: Vers des régimes d'asile plus accessible, équitables et organisés », Bruxelles, 3 juin 2003, $\operatorname{COM}(2003) 315$ final, p. 10.

96. La présente analyse est fondée sur des documents réunis, référencés ou publiés via Internet par deux organisations, au terme d'un travail collectif extrêmement précieux, notamment pour les chercheurs, de regroupement de données: Migreurop (http://pajol.eu.org) et statewatch (http://www.statewatch.org/).

97. Magner T., «A less than 'Pacific' Solution for Asylum Seekers in Australia », International Journal of Refugee Law, 2004, vol. 16, $\mathrm{n}^{\circ}$ 1, pp. 53-90 ; Papastergiadis N., « The invasion complex in Australian political culture ", Thesis Eleven, août 2004, n 78, pp. 8-27.

98. Commission européenne, "Communication from the Commission to the Council and the European Parliament: Towards a common asylum procedure and a uniform status, valid throughout the Union, for persons granted asylum », COM (2000) 755 final.

99. UNHCR, Agenda for protection, Geneva: UNHCR / Department of International Protection, octobre 2003 ( $3^{\text {ème }}$ ed.). 
100. UNHCR, «Statement by Mr. Ruud Lubbers, United Nations High Commissioner for Refugees, at an informal meeting of the European Union Justice and Home Affairs Council Copenhagen », 13 septembre 2002 .

101. Ibid., (notre traduction).

102. Peers S., "Statewatch Analysis: EU immigation and asylum discussions", Statewatch Observatory EU asylum and immigration policy. Voir: http://www.statewatch.org/asylum/ obserasylum.htm

103. D'après le Premier ministre britannique lui-même dans sa lettre à Costa Simitis. Pour une autre source, voir Gorlick B., HCR Stockholm (Suède), 16 avril 2004, in Bergström K., L'initiative Convention Plus du HCR: Vers une politique de régionalisation de la protection internationale des réfugiés?, Mémoire pour la Maîtrise de science politique, Dir. J. Valluy, Université Paris 1, septembre 2004, Annexes p. 21.

104. Deux articles du journal The Guardian: Travis A., "Shifting a problem back to its source Would-be refugees may be sent to protected zones near homeland ", The Guardian, 5 février 2003 ; Milne S., Travis A., " Safe havens plan to slash asylum numbers », The Guardian, 5 février 2003.

105. Chandler D., "Stopping asylum before it starts - The right to asylum in the UK is being transformed into the West's right to intervene abroad. », Spiked Liberties (revue en ligne), 19 février 2003.

106. Dans son discours du 18 mars 2003 devant la Commission des Nations Unies pour les Droits de l'Homme, Ruud Lübbers souligne la nécessité de trouver de nouvelles approches plus efficaces pour le management des flux migratoires.

107. « New international approaches to asylum processing and protection » (5 pages). Document accompagnant la lettre du 10 mars 2003 du Premier ministre britannique Tony Blair à son homologue grec assurant la présidence de l'Union européenne.

108. Ibid., traduction de la Confédération Française du Droit d'Asile (CFDA).

109. Conseil européen, «Communication...: Vers des régimes d'asile plus accessibles, équitables et organisés", op. cit., p. 3.

110. Migreurop, «Chronologie : De l'annonce du projet (mars 2003) au Sommet de Thessalonique (juin 2003)», 23 juillet 2002.

111. UNHCR, «Statement by Mr. Ruud Lubbers, United Nations High Commissioner for Refugees, at an informal meeting of the European Union Justice and Home Affairs Council, Veria, 28 March $2003 »$.

112. statewatch, « Asylum in the EU : the beginning of the end? ", mars 2003.

113. British Refugee Council, «Refugee Council briefing : position paper on the UK proposals for transit processing centres for refugees and regional management of asylum », Londres, mai 2003.

114. Amnesty International, «EU JHA Informal Council, Veria, 28-29 March: UK Refugee Proposals Legally Flawed Says Amnesty », 27 mars 2003.

115. Voir Prasad R., « Refugee camps don't work », The Guardian, 10 février 2003 et communiqué de presse ECRE, 17 mars 2003.

116. Source anonyme interne au HCR citée par le professeur Helmut Dietrich, «EUROPE : Camps de réfugiés aux nouvelles frontières extérieures. 2ème partie ", Archipel, 14 septembre 2003.

117. Amnesty International indique en avoir connaissance dès le mois d'avril. Voir A.I., « UK/EU/ UNHCR: Unlawful and Unworkable - Amnesty International's views on proposals for extraterritorial processing of asylum claims ", AI Index : IOR 61/004/2003, 18.06.2003, p. 2.

118. UNHCR, « UNHCR's three-pronged proposal », UNHCR Working Paper, Genève, juin 2003.

119. Cité in «'No plans' for asylum camps outside EU », The Guardian, 16 juin 2003.

120. Conseil européen, "Communication...: Vers des régimes d'asile plus accessibles, équitables et organisés ", p. 14.

121. "Justice et Affaires intérieures ", réunion des ministres européens de la Justice et de l'Intérieur. 
122. Spiteri S., « Asylum centres plan gains EU support », EUObserver.com, 06 juin 2003.

123. White M., Black I., "Go-ahead to experiment with Britain's refugee zones plan », The Guardian, 21 juin 2003.

124. Le ministre de l'Intérieur Otto Schily (Social démocrate) et le ministre des Affaires Etrangères Joschka Fischer (Verts) s'affrontent de manière récurrente sur les questions de l'asile. Voir notamment : « Germany calls to keep veto in immigration policy », EUObserver, Bruxelles, 02 juillet 2003.

125. Note interne : " Council of The European Union - Bruxelles 14 juin 2003 - 10462/03 LIMITE POLGEN 46 - From : The Presidency To : Council Subject : European Council (19/20 June 2003) Annotated draft agenda ».

126. Ibid., Paragraphe 26. Notre traduction. Le texte définitif sera différent.

127. " 'No plans' for asylum camps outside EU », Press Association, The Guardian, 16 juin 2003.

128. A.I., "UK/EU/UNHCR: Unlawful and Unworkable - Amnesty International's views on proposals for extraterritorial processing of asylum claims », op. cit.

129. FIDH / FIDH-AE, «Sommet de Thessalonique: Lettre ouverte aux chefs d'Etat et de Gouvernement des Etats Membres de l'Union européenne. ", Bruxelles, le 18 juin 2003.

130. A.I., «La politique d'asile commune de l'Union européenne : une perte de repères - Lettre ouverte aux chefs d'Etat et de gouvernement, Conseil européen de Thessalonique ", SF03R30 Bruxelles, 18 juin 2003 (trad. ASIF).

131. Conseil de l'Union européenne, « Note de transmission de : la présidence aux : délégations Objet: Conseil européen de Thessalonique 19 et 20 juin 2003 - Conclusions de la Présidence», Bruxelles, 01 octobre 2003, (OR. en) 11638/03 POLGEN 55.

132. Conseil européen de Thessalonique (19-21/6/03), Conférence de presse de la Présidence, 19 juin 2003.

133. Roche M., «Les Quinze s'efforcent de relancer leur politique d'immigration Londres ne renonce pas à des « centres » hors de l'UE », Le Monde, 21 juin 2003.

134. Grice A., Castle S., «Blair loses fight for 'refugee protection' zones but will push on », The Independent, 20 juin 2003.

135. White M., Black I., "Go-ahead to experiment with Britain's refugee zones plan », The Guardian, 21 juin 2003.

136. Lubbers R., «Put an end to their wandering Europe should do more to support refugees in their regions of origin », The Guardian, 20 juin 2003.

137. Addley E., « Welcome to camp Tirana It's been suggested that asylum-seekers arriving in the UK will be sent on to Albania. So how would they fare there ? ", The Guardian, 11 mars 2003.

138. Travis A., « New asylum centres open by end of year UK-bound refugees to be processed outside the EU ", The Guardian, 9 mai 2003.

139. Travis A., « Offshore asylum centres to be tested », The Guardian, 4 juin 2003.

140. Mahony H., « Bulgaria and Croatia refuse asylum camps » Euobserver.com, 16 juillet 2003.

141. "Germany calls to keep veto in immigration policy », EUObserver Bruxelles, 02 juillet 2003.

142. Discours du ministre de l'Intérieur G. Pisanu présentant le programme de la Présidence italienne en matière d'immigration, de criminalité organisée et de terrorisme, Bruxelles, 9 juillet 2003.

143. «Le HCR anticipe des problèmes possibles dans le système d'asile européen et propose des solutions », UNHCR Press Releases, 22 janvier 2004

144. UNHCR, "A revised 'EU Prong' proposal », UNHRC Working Paper, Genève, 22 décembre 2003.

145. EC, "Communication from the Commission to the Council and the European Parliement The Managed Entry in the EU of Persons in Need of international protection and the enhancement of the protection capacity of the region of origin - 'Improving access to durable solutions'", Bruxelles, $\operatorname{COM}(2004) 410$ final, 4 juin 2004. Pour un commentaire : ECRE, «Comments of the European 
Council on Refugees and Exiles on the Communication from the Commission ... ", CO2/09/2004/ ext/PC.

146. Statewatch / Hayes B., "Statewatch Analysis : Killing me softly? 'Improving access to durable solutions' : doublespeak and the dismantling of refugee protection in the EU », juillet 2004.

147. «Schily for EU Asylum Centers in Africa », Deutsche Welle, 20 juillet 2004.

148. AFP, «Un ministre propose la création de camps de demandeurs d'asile en Afrique », 21 juillet 2004 .

149. Le Tageszeitungde Berlin et la Rheinische Postde Düsseldorf s'interrogent sur les conditions de mise en œuvre, la Frankfurter Rundschau met en doute l'efficacité de la solution, la Süddeutsche Zeitung compare ce dispositif à l'ancien mur de Berlin. Voir Blanchard S., « Des camps de réfugiés en Afrique du nord?", Deutsche Welle, 21 juillet 2004.

150. Cité dans » Camps de demandeurs d'asile », L'Economiste (Maroc), 3 août 2004.

151. Reuters, « Rome et Berlin favorables à des 'portails' d'immigration », 13 août 2004.

152. "Immigration: initiative italo-allemande présentée au G5 en octobre", Tageblatt (Luxembourg), 14 août 2004.

153. «Des camps de demandeurs d'asile 'délocalisés'! - L'Afrique du Nord, terre d'accueil », L'Economiste (Maroc) 19 août 2004.

154. «Il Ministro degli esteri libico : Allarme da Tripoli 'Ormai siamo all'invasione' ", La Stampa, 9 août 2004, traduction assurée par Migreurop.

155. Mahony H., « EU faces immigration 'time-bomb' «, EuObserver, 23 août 2004.

156. Statewatch, « New Commissioner for 'Justice, Freedom and Security' talks of the EU being 'swamped' by a immigration 'time-bomb'«, 23 août 2004.

157. Joszsef E., «A Tripoli, Berlusconi croit enrayer l'immigration - Reçu par Kadhafi, il insiste pour ouvrir des 'centres d'accueil' en Libye » Libération, 26 août 2004.

158. Raaflaub C., Speziali B., «Blocher proposes setting up refugee camps abroad », Swissinfo, 28 octobre 2004.

159. Kirk L., "Four EU member states suggest refugee camp in Ukraine ", EU Observer, 16 septembre 2004.

160. "Ukraine dismisses notion of transit camps in the country ", EU Observer, 20 septembre 2004.

161. «EU to study transit sites in Libya for immigrants", International Herald Tribune, 24 septembre 2004.

162. « Débat confus sur la politique d'asile européenne », Le Monde, 01 octobre 2004.

163. Ibid.

164. UNHCR, « Talking points from video link address by Mr. Ruud Lubbers, United Nations High Commissioner for Refugees, to European ministers attending the Informal Justice and Home Affairs Council, Scheveningen, the Netherlands », Scheveningen, 1er octobre 2004.

165. Bouilhet A., «Union européenne Accord politique, hier, pour traiter les demandes d'asile à l'extérieur de l'Union l'Europe financera des 'centres' de réfugiés en Afrique du Nord ", Le Figaro, 02 octobre 2004.

166. Ibid.

167. « G5: pas de rapprochement en vue sur le projet de camps en Afrique du Nord », AFP, 17 octobre 2004.

168. Conseil de l'Union européenne, « Note de transmission de la : présidence, aux : délégations. Objet: Conseil européen de Bruxelles, 4 et 5 novembre 2004. Conclusions de la Présidence » Bruxelles, le 8 décembre 2004 (10.12) (OR. en) 14292/1/04 - REV 1. Annexe 1 : Le Programme de la Haye : Renforcer la liberté, la sécurité et la justice dans l'Union européenne ", pp. 11-42. « Note from: Presidency, to : Coreper/Council, Subject: Draft multiannual programme : «The Hague 
Programme ; strengthening freedom, security and justice in the European Union », Bruxelles, 15 octobre 2004, 13302/1/04, REV 1, LIMITE, JAI 370.

169. Statewatch / Steve Peers, "Statewatch annotation - updated The Hague Programme Strengthening freedom, security and justice in the EU doc no: 13302/2/04, 22 October draft ».

170. « Le Programme de la Haye », op. cit., p. 12.

171. Ibid., p. 13.

\section{RÉSUMÉS}

L'Europe politique offre une figure spécifique au regard des efforts actuels pour multiplier en son sein et chez ses voisins les dispositifs de regroupement forcé et d'enfermement des exilés (demandeurs d'asile, réfugiés, sans-papiers, clandestins...). La nouveauté réside moins dans l'existence de ces dispositifs que dans la facilité avec laquelle ils sont affichés dans l'espace public, comme instruments ou finalité de politiques publiques. Ceci révèle une transformation profonde des cultures européennes et de la gouvernance à l'égard des exilés, autrefois victimes à aider, aujourd'hui coupables menaçants. Hors cette convergence européenne, souvent imputée aux opinions publiques, passe par trois processus dont on peut montrer l'origine élitaire : la spirale du rejet des demandes d'asile, le tournant national sécuritaire à l'égard des étrangers et l'harmonisation européenne de la lutte contre l'immigration sous couvert de "justice, liberté, sécurité » (JLS). Les cultures politiques européennes se transforment ainsi sous l'effet de la montée en puissance d'une xénophobie de gouvernement.

The political Europe offers a specific figure if one considers the current efforts made to multiply within the Union and among its neighbours mechanisms of forced regrouping and confinement of exiles (asylum seekers, refugees, undocumented people, clandestine...). These systems are not new. What is new is the facility with which they are exposed in the public space as instruments and purpose to public policies. This reveals a profound transformation of the European cultures and of the governance in respect to exiles previously seen as victims in need of help and currently considered as threatening guilty people. But this European convergence, often imputed to the public opinion, goes through three processes having elite origins: the asylum rejection spiral, the national-security turn in respect to foreigners and to the European fight against immigration harmonization hidden by «justice, liberty, security » (JLS). The European political cultures thereby transform through the effect of the rise of government xenophobia.

\section{INDEX}

Mots-clés : Exilés, réfugiés, demandeurs d'asile, mise à l'écart, camps, justice, liberté, sécurité

\section{AUTEUR}

\section{JÉRÔME VALLUY}

Jérôme Valluy est Maître de Conférences en science politique à

l'Université Panthéon-Sorbonne (Paris 1), membre du Centre de Recherches Politiques de la 
Sorbonne (CRPS / CNRS), fondateur et co-animateur du réseau TERRA (Travaux, Etudes et Recherches sur les Réfugiés et l'Asile: http://terra.rezo.net). 